\title{
Le discours d'un Prince (Énéide, I, 597-610). Archéologie de la disposition
}

\section{Christine Noille}

\section{(2) OpenEdition}

1 Journals

Édition électronique

URL : http://journals.openedition.org/rhetorique/176

DOI : 10.4000/rhetorique. 176

ISSN : 2270-6909

Éditeur

UGA Éditions/Université Grenoble Alpes

Édition imprimée

ISBN : 978-2-84310-263-9

\section{Référence électronique}

Christine Noille, «Le discours d'un Prince (Énéide, I, 597-610). Archéologie de la disposition », Exercices de rhétorique [En ligne], 2 | 2013, mis en ligne le 28 janvier 2014, consulté le 12 septembre 2020. URL : http://journals.openedition.org/rhetorique/176 ; DOl : https://doi.org/10.4000/rhetorique.176

Ce document a été généré automatiquement le 12 septembre 2020.

\section{(c) (1) (2)}

Les contenus de la revue Exercices de rhétorique sont mis à disposition selon les termes de la Licence Creative Commons Attribution - Pas d'Utilisation Commerciale - Partage dans les Mêmes Conditions 4.0 International. 


\title{
Le discours d'un Prince (Énéide, I, 597-610). Archéologie de la disposition
}

\author{
Christine Noille
}

\section{Exorde}

Décembre 2012 : l'heure semble venue pour moi de devoir dire, en reprenant de loin Tristes Tropiques ${ }^{1}$, je hais les récits circonstanciés et les auteurs en mal de confidences, et voici que je m'apprête à raconter mon travail... Eh quoi ! Faut-il narrer par le menu tant de détails insipides, l'origine évanescente d'une idée, les aléas et les bifurcations de la pensée quand elle chemine à la poursuite d'une hypothèse ?... Et plus encore les oublis, les vides, les lenteurs de la conception, les pauses inévitables et au bout du compte, l'engourdissement de l'esprit et la difficulté à s'y remettre, à se ressaisir d'un projet théorique un peu long, un peu difficile... Aurais-je eu même le projet d'en faire le récit, une sorte de honte et de pudeur m'en aurait empêchée. Plus de vingt ans ont passé depuis mes premiers articles, et à chaque fois j'ai suivi le même procès, qui veut en effet que l'on oblitère les traces de la construction et que la façon d'exposer et de recomposer son sujet l'emporte haut la main en rigueur, en lisibilité, en cohérence, sur la façon de le trouver et de le débrouiller - tendus que nous sommes alors vers des fins de démonstration et de clarification.

Et qu'il faille tant de digressions et d'interpolations pour atteindre l'objectif de notre étude - une problématique stabilisée et partageable - ne confère cependant guère d'intérêt scientifique stricto sensu à cette accumulation préalable de notes et de contrenotes; tant il est vrai que nous ne retenons habituellement de tout cela que les seuls éléments entrant directement dans la structuration du débat que nous poursuivons et que nos travaux n'ont entre nous de portée que désencombrés de cette gangue de tergiversations et d'incidents de pensée.

C'est ainsi que pour ma part j'ai œuvré jusqu'ici à ma rhétorique de la dispositio. 
Quand, après quatre années passées à ausculter des pratiques rhétoriques anciennes passablement hermétiques et résistantes, j'ai eu fait l'hypothèse d'un point d'accroche possible et que dans la foulée j'ai eu identifié mon objet - je me souviens encore d'un matin où dans mon bureau à Grenoble, il y a deux ans, je l'ai dit comme en passant à Francis Goyet, «En fait je vais écrire sur la dispositio »; quand, cet objet, je l'ai eu délimité - non pas l'arrangement et les parties du discours, mais les gestes mêmes de composition syntagmatique, du discursif au narratif, du factuel au fictionnel; quand je l'ai eu réinterprété, articulé, conceptualisé - la disposition comme compétence de mise en forme, opération cognitive et affective de perception et d'identification des formes, structuration du texte; quand je l'ai eu maitrisé, je veux dire quand je l'ai rendu maîtrisable et maniable - ayant appris de l'ancienne rhétorique à distinguer trois grandes gestions de la disposition, par division, par ordonnancement, par enchaînement; quand je l'ai eu non pas uniquement théorisé, mais technicisé, mais formalisé - recensant méthodiquement, à partir de mes expérimentations textuelles et de mon innutrition des poéticiens contemporains, les dispositifs possibles de mise en forme pour chacune des trois catégories -, et que j'ai eu consigné dans une présentation détaillée l'ensemble de ma proposition technique sur ce qui était bel et bien devenu une rhétorique (lectoriale) de la disposition, le cheminement que j'y ai proposé n'était en rien celui de mon histoire de pensée. C'était un parcours dans la cartographie au net de ce à quoi j'avais abouti, c'était (pour reprendre une métaphore de webmaster ayant à gérer des cascades de pages numériques) comme un fil d'Ariane dans le déroulé de mes propositions finales.

5 C'est pourtant de là, de ce synopsis réel et attesté d'une vingtaine de feuillets, qu'il me faut aujourd'hui repartir - ou plutôt de sa réception, de ce qui fut au fond comme une péripétie : que mes premiers lecteurs - ceux-là même avec qui intellectuellement j'ai le plus d'affinités ${ }^{2}-\mathrm{y}$ lisent en miroir leurs propres pensées, y retrouvant approximativement les mêmes raisonnements, avec les mêmes hypothèses et selon les mêmes catégories... Un objet étrange, donc, comme s'il était un de ces hrönir passés du monde de Tlön dans le nôtre : un fragment coproduit par suggestion, un artefact déduit d'avoir été espéré. (Borges ajoute que le grand masque en or généré jadis lors d'une campagne de fouilles à l'endroit où tous le projetaient idéellement en est un autre exemple, illustre $\left.{ }^{3} . ..\right)$

6 A la réflexion (22 avril 2013), je pense qu'il est légitime de voir dans le synopsis final auquel je suis alors parvenue, ma propre contribution à ce qu'on pourrait appeler un programme de théorisation objectivement en voie de co-élaboration. J'ajoute que pour ma part je ne suis pas sûre qu'il soit à ce point ressemblant à la façon dont un Michel Charles ou un Francis Goyet pratiquent, d'ailleurs chacun à leur façon, la rhétorique et que dans le réseau formé par les déclinaisons que les uns ou les autres nous faisons par exemple de la dispositio, j'investis à ma façon les questions d'autonomisation et d'assemblage, de partition et d'ordonnancement, de séquençage et d'enchaînement.

7 Le champ est commun mais le discours que j'y développe - plus technique, plus pédagogique, plus politique - est de mon point de vue clairement identifiable, doté de ses marques propres. Encore faudrait-il sans doute rendre les rendre un peu plus visibles, un peu plus manifestes - non pas pour ma seule satisfaction, bien sûr, mais pour le projet en partage, pour ce qu'elles lui apportent en propre, en termes de redescription et de reformulation, en termes d'approfondissement et de déplacement. Aussi me permettrai-je de reprendre par un autre biais, plus saillant, moins familier, la 
déclinaison épistémologiquement spécifique que j’ai effectuée d'une théorie de la composition, en la replaçant dans son contexte d'émergence, autrement dit - nous y voilà - en la réinsérant dans son histoire : une histoire toute intellectuelle s'entend, l'histoire d'une expérience de compréhension, en prise avec des raisonnements particuliers et des questionnements, avec des suppositions, des vérifications, des décisions.

8 Tel sera donc le but de cette présente investigation. - Où l'on en passera bien par une archéologie de la dispositio pour en apprendre un peu plus sur la rhétorique du même nom.

\section{Proposition}

9 Ainsi donc, puisqu'il faut un commencement, ce sera, de la façon la plus archétypale, surgi des terres borgésiennes d'où il nous fait signe, le grand masque en or, le masque du Roi et avec lui, son pouvoir sur notre présent, sa capacité à nous interpeller, depuis toujours, sa force radicalement irruptive.

À l'instant le nuage s'ouvre et se dissipe, et Énée paraît.

À sa taille, à son air, on l'aurait pris pour un Dieu. Vénus elle-même a, d'un souffle de sa bouche, donné à ses cheveux une grâce nouvelle; elle a mis sur son visage l'éclat de la jeunesse et tempéré de douceur le feu de ses regards.

Tel brille l'ivoire, que la main de l'ouvrier a poli; et tels l'argent ou le marbre de Paros, quand ils se parent d'un reflet d'or.

Alors, s'avançant vers la Reine devant une assemblée médusée, le Prince dit ceci :

Le voici devant vous, celui que vous cherchez tous, le Troyen Énée, arraché aux flots de la Libye. Vous seule, Didon, prenant en pitié les grands malheurs de Troie, vous voulez bien nous recueillir, nous, les survivants du massacre grec, dépourvus comme nous le sommes de tout secours et épuisés par tous les fléaux de la terre et des mers, vous voulez bien nous recueillir dans votre cité, dans votre maison. Il n'est point en notre pouvoir, ô Reine, de vous remercier dignement; pas plus que ne le peut ce qui reste de la nation de Dardanus, dispersée par tout l'univers. Mais puissent les dieux, s'il en est qui honorent la vertu, s'il est quelque justice sur la terre, et s'il se trouve une conscience du bien, vous récompenser dignement. ô temps heureux qui vous ont vue naître! Quelle gloire pour les auteurs de votre naissance! Tant que les fleuves porteront leurs eaux à la mer, tant que les montagnes couvriront les vallées de leurs ombres, tant que les astres brilleront au firmament, je ne cesserai de proclamer votre gloire, votre nom et vos louanges, quelles que soient les terres où les destins m'appellent.

Le Prince ayant ainsi parlé, il tendit les bras à son ami Ilionée, à Sereste, puis aux autres Troyens, au vaillant Gyas et au vaillant Cloanthe $e^{4}$..

Voilà, tel qu'il a paru dans l'éclat d'un poème latin, le discours d'un Prince: relativement bref (quinze vers en v.o., douze lignes en v.f.) mais remarquablement dense, faisant défiler dans notre mémoire les débris de Troie et les malheurs des fugitifs, la grandeur de Carthage et la générosité de sa Reine, l'incandescence d'Énée et plus encore peut-être, l'offrande absolue de son âme dans ses paroles - tout un imaginaire de guerres et d'amours, de héros brisés et de dieux oubliant parfois d'être cruels.

Mais ce n'est pas tout.

12 Saisi dans le mouvement d'une intrigue (à la fin du chant I), ce discours est également un temps fort, une ponctuation clôturant l'exposition: il scelle la fin de la défiance 
carthaginoise face aux troupes troyennes naufragées et le début d'une alliance forte, politique puis sentimentale, entre la reine Didon et le chef des Troyens.

Replacé dans le contexte immédiat d'une scène de conciliation entre les deux peuples que le sort a mis en présence, il est pris dans un enchaînement de paroles, en amont la requête officielle des Troyens par la bouche de leur représentant, le vieil Ilionée - sollicitant de pouvoir rester en paix sur les rivages en attendant de repartir vers l'Italie -, ainsi que la réponse favorable de Didon, qui leur accorde l'hospitalité sur son territoire; en aval, la seconde réponse de Didon, qui les invite dans son palais. Ce qui fait, au bout du compte, une réponse de trop - au moins.

Et au milieu, donc, le discours du Prince: d'un côté, il répond à la faveur que Didon accorde aux Troyens par des remerciements en forme et évacue la séquence du naufrage ; de l'autre, il est un discours auquel il faut répondre, un discours d'appel qui rouvre l'intrigue. À la fois conclusion et commencement, réponse et proposition, il est en quelque sorte un discours charnière, occupant une position de transition et de basculement dans la syntaxe du récit comme dans la circulation de la parole. Mais ce n'est alors pas le moindre de ses paradoxes qu'aussi fortement intriqué dans le fil du texte, il ait été en même temps constamment autonomisé, extrait, cité - devenu un texte à part entière, auto-suffisant, un morceau de bravoure clos sur lui-même, un modèle offert à la mémoire et à l'imitation.

Car ce qui se joue dans l'espace textuel de ce discours a à voir avec la fabrique de l'exemplarité. Ou comment une séquence textuelle est prise en charge par un certain nombre d'opérations et de manipulations lectoriales, que la matérialité même du texte autorise et qui la travaillent en retour dans le sens du remarquage et de l'identification, du séquençage et de la focalisation. Ce processus de structuration et de modélisation porte un nom : c'est la rhétorique, du moins dans son versant à la fois le plus scolaire, le plus habituel, et le moins rappelé, longtemps ignoré des historiens comme des théoriciens de la chose ; la rhétorique en tant qu'elle est un art de lire tout autant qu'un art d'écrire, une pédagogie des compétences sollicitées dans la lecture tout autant qu'un compendium de procédés et de règles à l'usage des auteurs.

16 L'on sait en effet qu'il existe une abondante production d'ouvrages pédagogiques à usage des classes de rhétorique, qui, $\mathrm{du} \mathrm{xvI}^{\mathrm{e}}$ au $\mathrm{XIX}^{\mathrm{e}}$ siècle, ont servi de support à la lectio, à la leçon de rhétorique appliquée à l'analyse cursive des grands textes du répertoire humaniste, de Cicéron à Fléchier, de Tite-Live à Racine, de Rousseau (JeanBaptiste) à l'Énéide ${ }^{5}$. Autant de textes qui ont donné lieu à une expérimentation en situation des outils descriptifs de l'ancienne rhétorique et lui ont offert de préciser et de transmettre par l'usus, par l'usage, leur protocole d'application. Mais l'usage qui est alors exercé n'est pas d'abord celui de l'imitateur : c'est celui de l'attentif et suffisant lecteur, dont le regard (formé autant qu'informé) saura repérer les formes et figures de l'encyclopédie rhétorique dans le fil de la textualité. Un exercice de l'attention si l'on veut, de l'attention à la textualité qui est une attention à la fois formalisatrice et modélisatrice : la rhétorique (de la lecture) enseigne à remarquer dans les textes ce qu'il y a en eux de remarquable (pas très différente en cela de nos propres études de texte), en y disposant des marques qui vont permettre de s'y repérer et d'en favoriser la mémoire. Elle se révèle ainsi un exercice d'arpentage, œuvrant à analyser la composition du texte, à en décrire le plan (ce qu'elle nomme synopsis, argument, archétype) de façon à établir - à construire - à structurer sa lisibilité. Car la lisibilité est aussi (d'abord) affaire de structures ${ }^{6}$. 
17 Autrement dit, si dans les traités tournés vers la formation de l'orateur, la dispositio n'est qu'une des cinq parties de l'art, en revanche dans l'ancienne pédagogie rhétorique de la lecture, la dispositio est absolument centrale, mais elle devient bien autre chose qu'une méthode de présentation des arguments. Elle transmet un art de mettre en forme le texte en le lisant, un art d'identifier en lui des dispositifs susceptibles de le scander et de l'organiser, ou encore - et c'est là un saut épistémologique décisif - non pas un art de bien « voir » des dispositifs latents et en attente d'être vus, mais un art de formater le fil du texte à partir d'un certain nombre de dispositifs types, un art de motiver dans le texte un certain nombre d'énoncés comme embrayeurs de structuration et de modélisation, bref, redisons-le, un art de construire sa lisibilité en composant sa forme.

Et plus précisément, l'archéologie des gestes dispositionnels mobilisés par l'ancienne rhétorique quand elle se fait lecture, invite à distinguer et à maîtriser trois grandes gestions distinctes de la dispositio, de la mise en forme d'un texte ${ }^{7}$ :

- au niveau global de saisie du texte comme un tout auto-suffisant, une mise en forme par arrangement des séquences syntagmatiques en grandes parties, en s'aidant d'une bibliothèque des canevas discursifs : il s'agit là de penser les réseaux et les hiérarchies entre les séquences de façon à modéliser un ordonnancement d'ensemble susceptible de résumer le texte du discours et de le modéliser sur un des multiples discours type de l'encyclopédie rhétorique ${ }^{8}$;

- au niveau local de la focalisation sur la trame textuelle, une mise en forme par autonomisation et identification des séquences, en s'aidant d'une bibliothèque des formes élémentaires, essentiellement les formes d'arguments (les ressources argumentatives ou topoï ainsi que les formulations topiques), occasionnellement les formes d'expression (les figures ${ }^{9}$ ) : il s'agit là d'opérer des délimitations en distinguant des cellules dans le texte et en y reconnaissant des formes types, de façon à modéliser la division séquentielle du continuum syntagmatique ;

- au niveau du déroulé textuel enfin, une mise en forme par organisation du processus textuel dans le temps, en s'aidant d'une bibliothèque des transitions: il s'agit là de soumettre les hypothèses d'ordonnancement et de division à la prise en compte de la dynamique syntagmatique, du mouvement du texte, en interrogeant la présence et la pertinence des dispositifs de raccordement d'une séquence avec la suivante (niveau de la division) et des dispositifs d'enchainement des parties du canevas (niveau de l'ordonnancement).

19 Une telle gestion de la composition textuelle sollicite ainsi trois choses un peu différentes : la possession d'une bibliothèque des outils rhétoriques (elle-même adossée à une énorme encyclopédie d'« exemples»); la formation d'une compétence formalisatrice, d'une technique méthodique de structuration (par ordonnancement, division, transition); l'évaluation normative du texte composé, c'est-à-dire l'interprétation, l'ajustement que l'on peut faire entre les dispositifs de structuration identifiés et les formes modèles de l'encyclopédie. Le premier point relève d'un savoir culturellement déterminé, le deuxième d'une technique potentiellement actualisable, le dernier d'une herméneutique, d'une contextualisation des formes du texte. Et il se trouve précisément que le discours du prince Énée va devenir, par le biais des descriptions formalisatrices qui en sont faites et de l'interprétation normative qui les accompagne, un cas paradigmatique pour la rhétorique, le modèle exemplaire d'un type de discours, les remerciements. Ce qui sera la fois notre chance et notre souci. 
20 Notre chance, parce qu'il existe alors de nombreuses analyses qui en commentent la composition ou y réfèrent: et nous nous appuierons ici principalement sur les commentaires de trois rhétoriciens ${ }^{10}$, sans souci de leur ancrage chronologique ni institutionnel, celui de l'humaniste hollandais Gérard J. Vossius (1621), du recteur allemand Melchior Lauban (1610), du padouan Marco A. Ferrazzi, professant au séminaire de Padoue (1694). Mais ce sera également notre souci que ces trois commentaires, parce qu'avec la même volonté herméneutique d'en faire un discours exemplaire, la même encyclopédie des outils rhétoriques et a priori le même degré d'expertise dans la compétence formalisatrice, nos trois rhétoriciens ne structurent pas tout à fait de la même façon le texte de Virgile, de même qu'ils n'y reconnaissent pas tout à fait les mêmes formes modèles. Un subtil effet de décalage et de brouillage donc, mais qui nous permettra d'entrée de jeu de régler une difficulté majeure dans notre proposition de théorisation en faveur d'une rhétorique lectoriale de la disposition.

21 En effet, à la compréhension relativiste de la disposition que nous essayons ici de mettre en place, il est facile d'opposer une conception objective et matérielle, qui fasse des structures exemplifiées dans le texte non seulement un fait $d u$ texte, des propriétés possédées par le texte, mais encore des dispositifs exhibés (inévitables), parce que prescrits par l'intentio auctoris. Entre le projet initial et le texte finalisé, il y aurait comme le relais de structures saillantes dans le texte, qui seraient pertinentes en matière de modélisation et que le lecteur extérieur ne pourrait que remarquer.

Or, disons-le d'emblée, rien de plus vrai : il existe bel et bien des dispositifs textuels ostensiblement dédiés à une fonction de structuration, tels que, au niveau matériel, les sauts de page et en-têtes, les sauts de ligne et séparateurs, les alinéas, ou, au niveau sémantique, les récapitulations, les interpolations, les marqueurs logiques et chronologiques... Nous dirons même qu'ordinairement, dès lors qu'un texte est un peu long, ces dispositifs sont exubérants et qu'ils n'ont pas tous le même degré de pertinence: et l'on considèrera généralement qu'il $y$ a des prescripteurs de structuration plus saillants (tels que le chapitrage ou l'annonce introductive d'un plan) et des embrayeurs plus discrets (tels qu'un alinéa ou une formule du type « de plus ») ; des prescripteurs majeurs et d'autres subordonnés.

Mais le problème est bien là : devant la pléthore des dispositifs matériels et sémantiques susceptibles à la lecture d'être investis d'un fonctionnement structurant (c'est-à-dire pertinent en terme de séquençage, d'ordonnancement, de transition) - et devant l'irréductible liberté du lecteur, dont on sait bien depuis Paul Valéry, Umberto Eco et quelques autres ${ }^{11}$, qu'il ne remarque dans le texte que ce qu'il veut et que ce qu'il peut, indépendamment du programme lectorial que le texte lui prescrit -, il n'est pas possible d'en tenir pour une hiérarchie des structures fixée et latente, objective et intangible. La mise en forme du texte va s'opérer selon une constante négociation entre la prise en compte (ou non) des dispositifs de structuration surinscrits et l'investissement (ou non) des dispositifs discrets, selon un aménagement cognitif et affectif sans cesse révisé de la lisibilité du texte. Et la forme du texte sera très strictement la résultante de ce travail conjoint de remarquage et de formalisation qu'aura produit la lecture.

Ou pour le dire autrement, si tous les dispositifs possibles de mise en forme sont bien entés dans les énoncés du texte, sont bien permis par le fait du texte - et s'il est vrai qu'il doit être possible de les répertorier exhaustivement, par exemple grâce à une technique méthodique de recension (une rhétorique de la disposition, en d'autres 
termes) -, il n'en reste pas moins que tout texte, à chaque lecture, rejoue sa forme dans l'actualisation qu'il aura été possible d'en faire et qu'en conséquence, un certain nombre de textes deviennent méthodologiquement intéressants, soit qu'ils aient une disposition sur-inscrite et semblent imposer la forme de leur organisation - et l'on étudiera alors les dispositifs discrets de structuration susceptibles de relativiser les formes sur-inscrites et d'en prendre le relais; soit qu'ils n'aient pas de structuration saillante et donnent prise à des hypothèses de composition multiples - et l'on interrogera alors ce qu'il en est de la pertinence d'une hypothèse de division, d'ordonnancement ou d'enchaînement sur une autre.

Et nous revoilà précisément à ce discours de Prince, quelque part dans une poésie latine à l'orée du millénaire : car le discours d'Énée va être ainsi composé dans et par la lecture des rhétoriciens qu'à partir du même substrat sémantique (le matériel phrastique), nous allons avoir trois formes différentes du texte, trois textes devenus sensiblement différents par la gestion de leur lisibilité, de leur allure, de leur structure. Il n'est qu'à pousser jusqu'au bout la logique (visuelle) du plan que chaque analyse propose pour s'en rendre immédiatement compte, dès lors qu'on prend la peine de redistribuer le texte lu en distinguant par des sauts de ligne et des alinéas les parties et les sousparties :

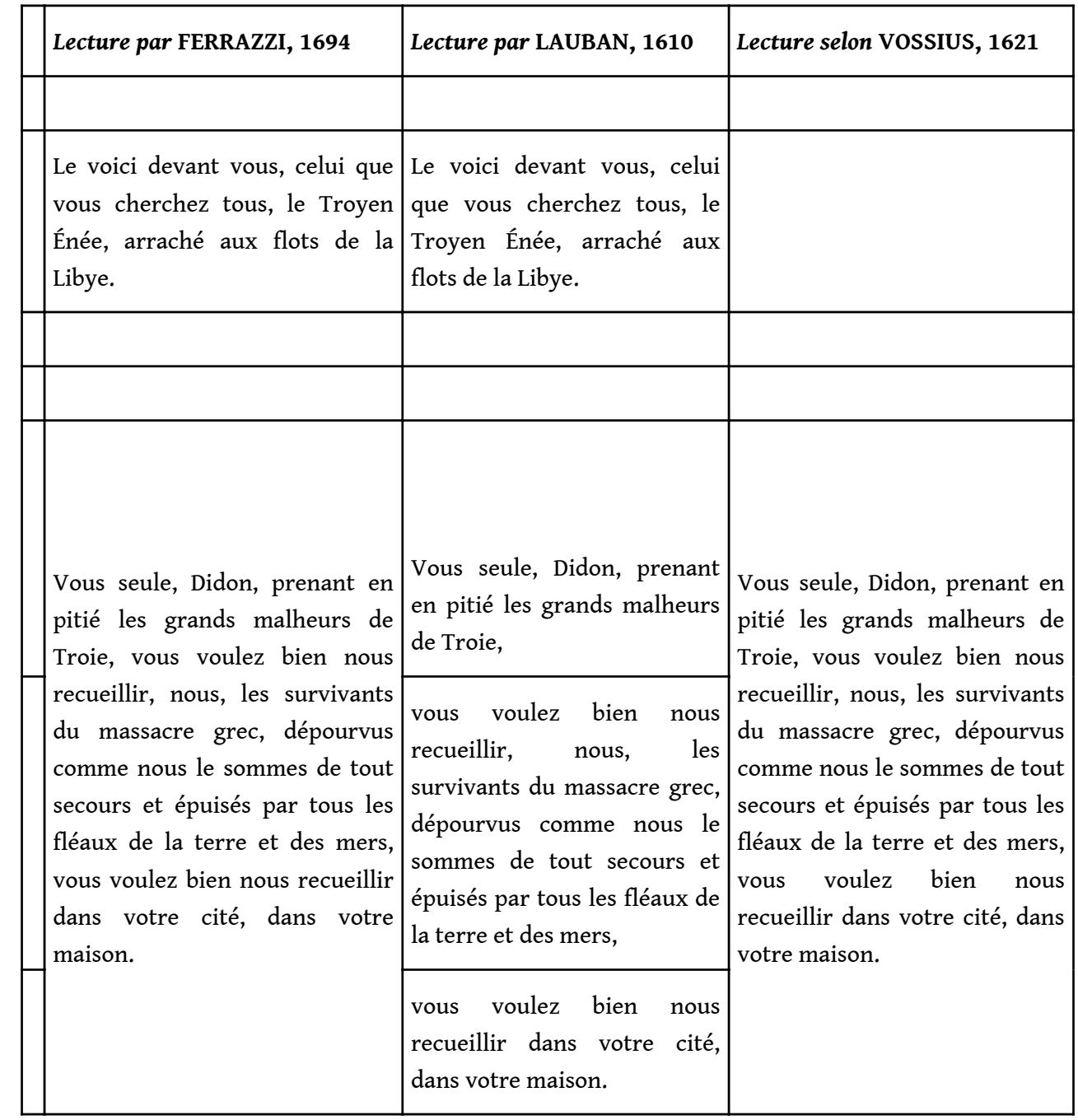




\begin{tabular}{|c|c|c|}
\hline $\begin{array}{l}\text { Il n'est point en notre pouvoir, ô } \\
\text { reine, de vous remercier } \\
\text { dignement; pas plus que ne le } \\
\text { peut ce qui reste de la nation de } \\
\text { Dardanus, dispersée par tout } \\
\text { l'univers. }\end{array}$ & $\begin{array}{l}\text { Il n'est point en notre } \\
\text { pouvoir, o reine, de vous } \\
\text { remercier dignement; pas } \\
\text { plus que ne le peut ce qui } \\
\text { reste de la nation de } \\
\text { Dardanus, dispersée par tout } \\
\text { l'univers. }\end{array}$ & \\
\hline \multirow[t]{2}{*}{$\begin{array}{l}\text { Puissent les dieux, s'il en est qui } \\
\text { honorent la vertu, s'il est } \\
\text { quelque justice sur la terre, et } \\
\text { s'il se trouve une conscience du } \\
\text { bien, vous récompenser } \\
\text { dignement. }\end{array}$} & & $\begin{array}{l}\text { Il n'est point en notre pouvoir, } \\
\text { ô reine, de vous remercier } \\
\text { dignement; pas plus que ne le } \\
\text { peut ce qui reste de la nation } \\
\text { de Dardanus, dispersée par } \\
\text { tout l'univers. }\end{array}$ \\
\hline & $\begin{array}{l}\text { Puissent les dieux, s'il en est } \\
\text { qui honorent la vertu, s'il est } \\
\text { quelque justice sur la terre, } \\
\text { et s'il se trouve une } \\
\text { conscience du bien, vous } \\
\text { récompenser dignement }\end{array}$ & $\begin{array}{l}\text { Puissent les dieux, s'il en est } \\
\text { qui honorent la vertu, s'il est } \\
\text { quelque justice sur la terre, et } \\
\text { s'il se trouve une conscience du } \\
\text { bien, vous récompenser } \\
\text { dignement. }\end{array}$ \\
\hline $\begin{array}{l}\text { Ô temps heureux qui vous ont } \\
\text { vue naître! Quelle gloire pour } \\
\text { les auteurs de votre naissance! }\end{array}$ & $\begin{array}{l}\text { Ô temps heureux qui vous } \\
\text { ont vue naître! Quelle gloire } \\
\text { pour les auteurs de votre } \\
\text { naissance! }\end{array}$ & $\begin{array}{l}\text { Ô temps heureux qui vous ont } \\
\text { vue naître! Quelle gloire pour } \\
\text { les auteurs de votre naissance! }\end{array}$ \\
\hline $\begin{array}{l}\text { Tant que les fleuves porteront } \\
\text { leurs eaux à la mer, tant que les } \\
\text { montagnes couvriront les } \\
\text { vallées de leurs ombres, tant que } \\
\text { les astres brilleront au } \\
\text { firmament, je ne cesserai de } \\
\text { proclamer votre gloire, votre } \\
\text { nom et vos louanges, quelles que } \\
\text { soient les terres où les destins } \\
\text { m'appellent. }\end{array}$ & $\begin{array}{l}\text { Tant que les fleuves } \\
\text { porteront leurs eaux à la } \\
\text { mer, tant que les montagnes } \\
\text { couvriront les vallées de } \\
\text { leurs ombres, tant que les } \\
\text { astres brilleront au } \\
\text { firmament, je ne cesserai de } \\
\text { proclamer votre gloire, votre } \\
\text { nom et vos louanges, quelles } \\
\text { que soient les terres où les } \\
\text { destins m'appellent. }\end{array}$ & $\begin{array}{l}\text { Tant que les fleuves porteront } \\
\text { leurs eaux à la mer, tant que } \\
\text { les montagnes couvriront les } \\
\text { vallées de leurs ombres, tant } \\
\text { que les astres brilleront au } \\
\text { firmament, je ne cesserai de } \\
\text { proclamer votre gloire, votre } \\
\text { nom et vos louanges, quelles } \\
\text { que soient les terres où les } \\
\text { destins m'appellent. }\end{array}$ \\
\hline
\end{tabular}

Que constate-t-on d'emblée ? D'abord que le texte ne commence pas pour tous au même endroit; ensuite qu'il est généralement divisé en deux grandes parties, mais que nul ne s'accorde sur leurs délimitations respectives; enfin qu'il est subdivisé ici et là en un certain nombre de séquences élémentaires relativement stables d'une analyse à l'autre. Autrement dit, il semble qu'il y ait accord sur la division séquentielle et sur l'ordonnancement en deux parties, mais pas sur les lieux frontière (incipit ou transitions); ou qu'il y ait un certain degré d'automatisme et partant un relatif consensus dans le séquençage et l'organisation générale, mais que la précision des formes (de leurs contours) passe par leur identification (leur contextualisation dans l'encyclopédie rhétorique des formes types) et partant par leur interprétation. Si la formalisation relève d'une technique, l'hypothèse de modélisation engage aussi une 
herméneutique : c'est là un point épistémologiquement intéressant, assurément, et nous y reviendrons. Mais l'essentiel n'est peut-être pas là. Il est d'abord dans la pédagogie de tous ces gestes techniques qui ont permis ces mises en forme concurrentes et que nous allons expérimenter en même temps que nous essaierons d'en rendre compte. Ce qui revient à dire que la méthode d'investigation cesse ici pour nous d'être une archéologie, pour s'avérer une initiation au présent, dans notre présent, l'apprentissage en situation de cette technique de lecture qu'est la rhétorique de la dispositio.

Car il n'est d'ancienne rhétorique que maintenue à distance comme objet d'un savoir ; à partir du moment où l'on bascule du côté des savoir-faire, on entre dans l'actualité d'une pratique méthodique. Et la rhétorique (re)devient une proposition.

\section{Vossius ou les remerciements en forme}

\section{Éléments d'analyse pour un discours de remerciement}

L'ouvrage de Vossius Rhetorices Contractae (que nous nous faisons un devoir de traduire par Rhétorique restreinte $e^{12}$ ) n'est pas un recueil d'analyses rhétoriques, mais un traité à usage des orateurs, dont un des chapitres porte (entre autres) sur le discours type des remerciements en forme. Simplement, la conjointure entre art d'écrire et art de lire est à ce point prégnante, que les préceptes sont très systématiquement entrelardés d'exemples tantôt cités et tantôt mentionnés (car il est inutile et impossible à la fois de mettre un discours de Cicéron en entier : c'est trop long et c'est archi-connu, on ne mettra donc que les premières phrases initiales de l'endroit exemplifiant la règle - comme en musique les premières notes suffisent à donner l'air).

Les recommandations pour composer un discours de remerciement s'étendent sur sept items numérotés ( $\left.n^{\circ} 2-8\right)$, avec distinction de deux discours types, les remerciements directs et les remerciements indirects (définition donnée dans l'item $n^{\circ} 2$ ), ces derniers étant traités brièvement à la fin (item $n^{\circ} 8$ ). L'essentiel de l'analyse porte sur les remerciements directs (items $n^{\circ} 3-7$ ), avec le détail du canevas type (items $n^{\circ} 4$ à 7 ) et une série d'exemples tantôt analysés, tantôt simplement donnés à l'attention et à la mémorisation (long développement inséré après l'item nº 7) : 


\section{G. J. VOSSIUS, Rhetorices Contractae [Rhétorique Restreinte]

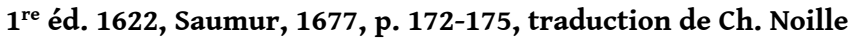

\section{Discours de remerciement [extraits]}

[...] 2. LE DISCOURS DE REMERCIEMENT est ou bien direct; ou bien indirect.

3. Est direct le discours dans lequel, ouvertement, sans un quelconque circuit, nous disons merci.

4. Il comporte trois parties. Dans la première, nous signifions la joie que nous a procurée le bienfait reçu.

5. Dans une autre partie, nous amplifions ce bienfait, de sorte qu'il apparaisse visiblement que nous comprenons sa grandeur. C'est en effet une qualité supérieure pour celui qui est reconnaissant, que de savoir combien il doit et de le devoir de bon gré et sans réticence.

Quand nous nous y prenons ainsi pour amplifier le bienfait, nous montrons très visiblement que nous le faisons sous l'effet de la gratitude.

6. La troisième partie promet la gratitude. Ce qui peut se faire de deux façons. Car soit nous disons que nous témoignerons en retour d'une gratitude tout aussi grande, voire supérieure ; soit, si c'est au-dessus de nos forces, à tout le moins nous nous engageons solennellement à être reconnaissants et à nous souvenir du bienfait: et nous prions Dieu de répandre lui-même toutes les grâces qui conviennent, étant donné que nous en sommes au plus haut point incapables, vu nos faibles moyens.

7. A tout cela s'ajoute parfois une autre recommandation, dans laquelle nous nous permettons la requête suivante: que celui qui nous a touchés par ce bienfait, veuille bien nous combler de son amour. Et pareillement, nous pouvons formuler un vœu : que Dieu veille à son salut.

Comme exemple de ce genre de discours, on peut prendre deux discours de Cicéron : l'un prononcé après son retour devant les Citoyens [Post reditum ad Quirites, C. U. F. vol. 13-1] ; l'autre après son retour au Sénat [Post reditum in Senatu, ibid.]. [...]

C'est de la même façon qu'est construit le second discours:

(I). Il proteste de sa gratitude pour le bienfait reçu et il le dit avec force.

(II). Il se lance dans une invective contre ceux qui, par jalousie, causèrent son éviction.

(III). Il célèbre le bienfait dont ses amis l'ont comblé.

(IV). Il promet de se dévouer à la République.

Autre exemple semblable, le discours de Cicéron dans lequel il remercie César d'avoir rappelé Marcellus [Pro Marcello, C. U. F. vol. 18]. Autre exemple illustre, le discours d'Énée à Didon :

Vous seule, Didon, prenant en pitié les grands malheurs de Troie, vous voulez bien nous recueillir, nous, les survivants du massacre grec, dépourvus comme nous le sommes de tout secours et épuisés par tous les fléaux de la terre et des mers, vous voulez bien nous recueillir dans votre cité, dans votre maison. Il n'est point en notre pouvoir, ô reine, de vous remercier dignement ; pas plus que ne le peut ce qui reste de la nation de Dardanus, dispersée par tout l'univers. Puissent les dieux, s'il en est qui honorent la vertu, s'il est quelque justice sur la terre, et s'il se trouve une conscience du bien, vous récompenser dignement. Ô temps heureux qui vous ont vue naître ! Quelle gloire pour les auteurs de votre naissance! Tant que les fleuves porteront leurs eaux à la mer, tant que les

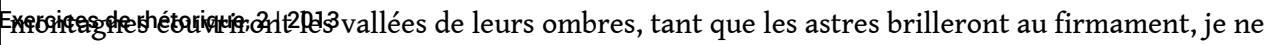
cesserai de proclamer votre gloire, votre nom et vos louanges, quelles que soient les terres où les destins m'appellent. 
31 façon générale dans ces répertoires de types de discours, la simplicité discursive n'est pas celle du déroulé syntagmatique, elle est celle du contexte pragmatique d'énonciation, et plus précisément elle définit à la fois l'éthos de l'orateur (l'orateur peut et veut énoncer le discours), celui du destinataire (le destinataire peut et veut l'entendre) et, le cas échéant, l'éthique du propos (la chose peut être dite sans contrevenir à ce qui est juste, bon, utile). dire des énoncés prenant en charge un travail sur le contexte éthique. Mais ils ne font pas l'économie des autres formes, et en l'occurrence plusieurs règles de composition sont mises en avant. Les décrire nous permettra au moins deux choses, et peut-être trois : nous aurons une idée de ce qu'est une règle d'écriture dans la mise en place d'un discours type; nous aurons une idée de ce qu'est un discours de remerciement; et peut-être irons-nous jusqu'à mettre les règles en situation (sinon de production, du moins de lecture) : mais il est vrai que nous serons alors aidés par les développements de Vossius sur les exemples.

\section{Protocole de l'analyse}

L'analyse repose ici sur trois outils clef de l'encyclopédie rhétorique, qui sont autant de formes (au sens de moulages, de gabarits pour mettre en forme) servant à décrire comme à prescrire quelque chose du texte : le propositum, les partes, les loci $i^{13}$. C'est ce savoir ancien, historiquement situé, conceptuel avant que technique, qu'il nous faut mettre en place méthodiquement pour pouvoir ensuite avancer dans la compréhension (dans l'actualisation) des gestes dispositionnels.

Très clairement, tout d'abord, la régulation d'un type de discours porte longuement sur son canevas, ce qui revient à dire que la définition d'un type de discours est tout autant syntagmatique que pragmatique. Et plus précisément, dans l'analyse que Vossius consacre aux remerciements directs, il nous est principalement prescrit un plan, en quatre parties.

Mais il est à noter un point essentiel quoiqu'implicité (tant il est évident) : cette partition dépend en amont d'un item introducteur qui à la première lecture semble une définition un peu tautologique du discours de remerciements («Est direct le discours dans lequel [...] nous disons merci »). Or, bien sûr, il n'en est rien. Il s'agit là aussi d'une prescription, capitalissime, qui engage à la fois le genre du discours (niveau paradigmatique) et l'arrangement de ses parties (niveau syntagmatique). En effet, la locution verbale utilisée ("gratias agere ») met en avant deux sèmes, s'activer à un but et donner des marques de gratitude, de reconnaissance. Plutôt que la traduction stéréotypée que nous avons initialement proposée, il est donc possible de comprendre très strictement: «Est direct le discours dans lequel [...] nous protestons de notre gratitude, nous donnons l'assurance de notre reconnaissance ». Ce qui signifie que les remerciements ne sont pas à énoncer, ils sont à prouver ; le destinataire ne fait pas que les recevoir : il doit y croire. Nous retrouvons là une pièce centrale dans le dispositif encyclopédique de l'ancienne rhétorique, sa vocation à prendre en charge l'argumentation persuasive, autrement dit (si l'on passe du côté de la réception), sa décision de postuler l'argumentativité des énoncés qu'elle prend en charge, d'interpréter toute parole comme production d'un argumentaire en vue d'un but. Telle est la raison pour laquelle, 
avant le plan, est prescrit ce à quoi œuvre tout discours de remerciement, ce qu'il se propose de prouver (sa "proposition", son propositum, dans les termes de l'art), ce sur quoi - tout aussi bien - il aura à argumenter.

Et les quatre "parties » du plan sont alors autant de dispositifs susceptibles d'établir l'assurance de ma gratitude : 1 . parce que je suis tout à la joie de la faveur qui m'a été accordée, 2. parce que je n'en reviens pas de sa grandeur, 3. parce que je me confonds en protestations de gratitude, 4. parce que j'en viens même à penser que tout m'est offert - que mon bienfaiteur peut m'aimer - que mon Dieu peut l'aimer -, tous mes propos ne démontrent qu'une chose, la profondeur, la sincérité de mes remerciements. Autrement dit, les «parties» du discours sont des éléments probatoires (des " preuves») tout autant que des éléments dispositionnels; ou plus exactement, elles sont la projection, sur l'axe de la disposition, de la construction argumentative. Le système du discours repose ainsi sur la liaison entre proposition et parties, entre argumentaire et canevas.

Mais outre la fonction, il y aussi la forme : le canevas type ne se contente pas de définir les parties par leur fonction dans l'édifice probatoire, il décrit également leur extension, en énumérant les arguments susceptibles de les composer. Ce qui veut dire que chacune des quatre "parties» (des quatre blocs argumentatifs) étant dédiée à la démonstration d'un élément probatoire dans l'assurance des remerciements (établir la joie que nous avons ; établir la grandeur du bienfait ; établir la promesse de gratitude ; établir notre foi en l'autre), elle peut être elle-même subdivisée en arguments types susceptibles de la constituer (niveau syntagmatique) et de l'établir (niveau paradigmatique). La même organisation qui prévaut au niveau global et principal est redupliqué au niveau local et subordonné. Le discours type est un dispositif modulaire complexe, parfaitement intégré.

Et si l'on passe enfin au niveau des arguments types entrant dans la composition et la probation des parties, sont alors attestées dans les traités deux descriptions possibles des arguments, une description formelle (décrivant la forme de l'argument, ce dont il tire sa vis persuadendi, le topos ou locus communis à partir duquel il s'établit : argument $a$ causa, a circumstantiis, a comparatione, etc.) ; et une description sémantique (décrivant le contenu argumentatif en en donnant une formulation sommaire, en en énonçant le locus spécifié). C'est cette dernière option qui prévaut ici, comme on le voit pour la " troisième partie ", consacrée à la promesse de gratitude (règle $\mathrm{n}^{\circ} 6$ ). Deux arguments sont résumés pour établir la gratitude : il faut promettre une gratitude (au sens actif de grâce à accorder ${ }^{14}$ ) répondant à la faveur qui nous comble ; ou bien il faut énoncer que notre gratitude ne pourra jamais être à la hauteur d'un tel bienfait. Ce dernier cas est particulièrement intéressant, et dans la démarche de Vossius, et pour le texte d'Énée, que nous n'avons pas tout à fait perdu de vue :

[...] Si c'est au-dessus de nos forces, à tout le moins nous nous engageons solennellement à être reconnaissants et à nous souvenir du bienfait : et nous prions Dieu de répandre lui-même toutes les grâces qui conviennent, étant donné que nous en sommes au plus haut point incapables, vu nos faibles moyens.

L'on voit ici au passage combien le canevas type est proche du discours type : il décrit sa composition jusqu'à un niveau de granularité fine, tout en donnant une formulation littérale de ses éléments. Mais on peut en déduire la vérité inverse : chaque formulation (chaque énoncé, chaque séquence syntagmatique) a valeur de composante fonctionnelle dans le cadre auquel elle se raccroche. Ce qui veut dire qu'il convient d'avoir une approche à la fois dispositionnelle et fonctionnelle de chaque formule. On 
peut alors diviser l'énoncé rappelé à l'instant en trois éléments fonctionnellement interdépendants et structurellement ordonnés :

- 1. « C'est au dessus de nos forces » que de rendre la pareille, micro-proposition cardinale de la cellule, qui est reprise en clôture par une formule de récapitulation : « Nous en sommes au plus haut point incapables, vu nos faibles moyens ";

-2. Conséquence principale de la micro-proposition, « Nous nous engageons solennellement à être reconnaissants et à nous souvenir du bienfait »;

-3. Conséquence en second, «Nous prions Dieu de répandre lui-même les grâces qui conviennent ", c'est-à-dire de payer notre dette de reconnaissance par un bienfait à la hauteur de celui que nous avons reçu.

C'est bien évidemment à ce moment-ci de la compréhension du protocole que surgit pour nous la possibilité (la tentation) de ne pas en rester là, à ce niveau à peu près satisfaisant de conceptualisation théorique, de reconstitution du meccano discursif dans l'analyse rhétorique, mais de basculer dans sa mise en pratique, dans son application à la lecture d'un texte, et partant dans son expérimentation. Décision inaugurale, qui non seulement nous mettra en prise avec la mise en œuvre méthodique d'une pratique de lecture, mais transformera les quelques outils d'analyse discursive que nous avons répertoriés en outils cognitifs, en relais de notre savoir-lire. C'est ce que nous pourrions appeler la tentation du bricoleur : une proposition, quatre parties, une dizaine de lieux argumentés, voyons comment ça marche. Heureusement que le discours d'Énée est là pour cela - pour être lu.

\section{Réfutabilité des hypothèses}

Résumons : d'un côté, nous avons les règles des remerciements en forme. De l'autre, nous avons les exemples proposés - car Vossius n'en reste pas à la théorie, il l'ancre immédiatement dans l'analyse d'un certain nombre de textes. Deux discours de Cicéron sont référés et analysés (le Post reditum ad Quirites et le Post reditum in Senatu, dont nous avons seule conservé ici l'analyse) ; un autre est mentionné comme exercice possible (le Pro Marcello) ; et, cerise sur le gâteau, un discours - plus bref, plus dense - est cité sans analyse, mais in extenso : ce sera donc le discours d'Énée.

Premier point pédagogique somme toute simple et évident, les exemples convoqués sont de droit des exemples entrant dans le genre des remerciements : Cicéron, tout comme Énée, a bénéficié d'une faveur (son retour à Rome) et il a également un intérêt politique à persuader l'assemblée des citoyens ou le Sénat de sa gratitude. Et nulle complication éthique ne vient inciter à développer des précautions oratoires.

Deuxièmement, puisque le canevas type des remerciements directs est focalisé sur quatre parties très identifiées (du point de vue fonctionnel comme du point de vue de la formulation), il est possible d'aborder chaque texte exemplaire en essayant de les y reconnaître - autrement dit en formulant une hypothèse sur sa construction, en décidant de modéliser son plan. C'est ce que fait d'emblée Vossius quand il analyse par exemple le second discours de Cicéron. Sans que nous ayons (dans notre culture, dans notre bibliothèque...) le support textuel susceptible de nous en apprendre un peu plus sur les raisons des délimitations et la pertinence des résumés, mais en faisant crédit à Vossius de la validité de son analyse, une petite inquiétude se fait jour malgré tout : les quatre parties qu'il spécifie ne correspondent pas aux quatre parties d'un discours de remerciement. 


\begin{tabular}{|c|c|}
\hline $\begin{array}{l}\text { Analyse par Vossius } \\
\mathrm{du} \text { Post reditum in Senatu } \\
\text { (Cicéron) }\end{array}$ & $\begin{array}{l}\text { Canevas du discours type de remerciement } \\
\text { selon Vossius }\end{array}$ \\
\hline $\begin{array}{l}\text { (I) "Il [Cicéron] proteste de sa } \\
\text { gratitude pour le bienfait reçu et il le } \\
\text { dit avec force." }\end{array}$ & $\begin{array}{l}\text { A. « Nous signifions la joie que nous a procurée le bienfait } \\
\text { reçu." }\end{array}$ \\
\hline $\begin{array}{l}\text { (II) "Il se lance dans une invective } \\
\text { contre ceux qui, par jalousie, causèrent } \\
\text { son éviction." }\end{array}$ & $\begin{array}{l}\text { B. « Nous amplifions ce bienfait, de sorte qu'il apparaisse } \\
\text { visiblement que nous comprenons sa grandeur." }\end{array}$ \\
\hline $\begin{array}{l}\text { (III) "Il célèbre le bienfait dont ses } \\
\text { amis l'ont comblé." }\end{array}$ & C. « La troisième partie promet la gratitude. " \\
\hline $\begin{array}{l}\text { (IV) «Il promet de se dévouer à la } \\
\text { République.» }\end{array}$ & $\begin{array}{l}\text { D. «A tout cela s'ajoute parfois une autre recommandation } \\
{[\ldots] \text { : que celui qui nous a touchés par ce bienfait, veuille bien }} \\
\text { nous combler de son amour. Et pareillement }[. . .] \text { : que Dieu } \\
\text { veille à son salut.» }\end{array}$ \\
\hline
\end{tabular}

Disons pour aller vite qu'il n'est pas prévu, dans le canevas type, qu'une partie puisse être dévolue à l'invective comme ici la partie II ; ni que la promesse de gratitude puisse s'étaler sur deux parties et exploiter les deux manières, promesse de reconnaissance (en I) et promesse de rendre la pareille (en IV); ni que la partie inaugurale (signifier sa joie) puisse être déplacée (en III). Et pourtant Vossius, qui, d'un côté, connaît son plan type, qui, de l'autre, a distingué parmi tant d'autres le Post reditum in Senatu comme un discours exemplaire du type des remerciements, est finalement capable de le séquencer sans se livrer à une application standardisée de l'outil que constitue le canevas type pour repérer, identifier et délimiter les parties du discours.

Bref, il s'agit incontestablement là d'une expertise et d'une agilité dans l'art de mettre le texte en forme, d'une application non mécanique : nous en resterons quant à nous à un objectif plus humble dans un premier temps, l'application «scolaire ", littérale, du canevas-type au discours d'Énée. Ce qui veut dire aussi qu'il n'y a pas lieu de vilipender un usage simple et non critique de la technique, de la méthode de partitionnement : de fait, une solidarité profonde existe entre une pratique lectoriale débutante et une pratique experte, toutes deux mobilisant une même compétence cognitive somme toute très élaborée, la mise en forme de cet objet immatériel s'il en est qu'est la textualité. C'est bien d'ailleurs ce que nous allons éprouver pour notre propre compte, en parcourant la gamme qui va de la phase d'apprentissage à la phase d'automatisme, puis à la phase critique et à la phase de reformulation ${ }^{15}$. Mais il faut un début à tout.

Et pour en revenir au discours d'Énée, reconnaissons que nous ne sommes (presque) pas aidés par le lecteur expert Vossius. Il ne fait, somme toute, que l'essentiel : nous le donner comme exemplaire (autrement dit nous signifier que sa proposition argumentative est par excellence celle d'un discours de remerciement, à nous qui savons désormais qu'il existe un genre discursif dévolu aux remerciements en forme) et nous (re)mettre ensuite sous les yeux le texte littéral, sans indication concernant sa composition. À une exception près cependant, et de taille : il évacue la première phrase, 
pourtant si énergique avec son entame par un adverbe («Coram, quem quaeritis... », « Le voici devant vous, celui que vous cherchez tous... ») et refait en quelque sort le début du discours en le faisant commencer sur l'incantation à Didon " 0 sola...", "Vous seule, Didon... ». Disons après coup que l'une et l'autre formule sont également des énoncés potentiellement embrayeurs d'un commencement ${ }^{16}$, mais que Vossius semble identifier deux ensembles, une grande masse qu'il associe au genre et à la visée argumentative des remerciements en forme, et une séquence qu'il détache purement et simplement parce qu'extérieure au propos. Un rhétoricien et philologue que nous recroiserons, le jésuite espagnol Juan Luis de La Cerda, identifiait ainsi le décrochage :

Les paroles qui précèdent s'adressent à tous : celles qui suivent, à la seule Didon ${ }^{17}$

[...].

Très clairement, pour des raisons fondamentales (situation d'interlocution, visée argumentative, genre de discours...), il est possible d'isoler et d'autonomiser la séquence initiale hors du système des remerciements. Du point de vue méthodologique, non seulement nous vérifions une fois encore qu'il existe dans un discours des séquences interpolées par rapport au canevas type, des séquences qui n'entrent pas dans le plan ni dans le genre: dans le second discours de Cicéron, Vossius l'identifiait sans autre commentaire («(II) Il se lance dans une invective contre ceux qui, par jalousie, causèrent son éviction ») ; dans le discours d'Énée, sans doute parce qu'elle est en marge, elle est évacuée. Mais nous apprenons aussi qu'il convient de reposer à chaque séquence la question du propositum, du dessein, la question de son intégration (ou non) dans la proposition d'ensemble.

Donc, il nous reste, pour avancer dans notre apprentissage des gestes de mise en forme, tout l'ensemble ouvert par l'interpellation à Didon. Nous pouvons alors risquer une négociation point par point entre le canevas type selon Vossius et notre texte. Munis des formules identifiant les grandes parties, les sous-parties et les micro-séquences, nous allons essayer de les repérer dans le tissu du texte, mutatis mutandis, c'est-à-dire avec la marge d'adaptation et de variation qui fait du processus de reconnaissance une opération herméneutique tout autant qu'un séquençage structurant.

1. Des quatre parties résumées dans le tableau que nous venons de donner un peu plus haut, rappelons que les trois premières sont exposées sans condition (valables en toutes circonstances), tandis que la dernière est une possibilité : selon les circonstances, nous pouvons nous recommander à l'amour de notre bienfaiteur ou le recommander à l'amour de Dieu. Il est alors aisé de constater que cette séquence est absente du texte.

50 2. La première partie du canevas est elle aussi passablement étrangère au texte retenu : nulle formulation qui aille dans le sens de la laetitia, qui expose un intense sentiment de joie et en rende raison. Le discours d'Énée, à l'incipit, met bien plus en scène les ressorts d'un pathos inverse, la pitié, à travers le tableau qu'il donne des misères endurées par les Troyens.

51 3. Restent les parties dévolues à l'amplification du bienfait et à la promesse de gratitude. Énée montre-t-il qu'il comprend la grandeur du bienfait? Et se montre-t-il soucieux de payer sa dette de reconnaissance? La réponse est manifestement positive dans les deux cas. Nous dirons que nous pouvons modéliser notre description du texte sur les impératifs du canevas type, en disant par exemple : d'une part, Énée appuie la grandeur du bienfait par comparaison avec la misère du peuple troyen et d'autre part, il n'en finit pas de se perdre en promesses de reconnaissance éternelle. Et du même coup, il est possible d'introduire une délimitation et une répartition des énoncés. Le 
locus synoptique « Nous amplifions ce bienfait, de sorte qu'il apparaisse visiblement que nous comprenons sa grandeur » trouve écho dans la grande formule initiale :

Vous seule, Didon, prenant en pitié les grands malheurs de Troie, vous voulez bien nous recueillir, nous, les survivants du massacre grec, dépourvus comme nous le sommes de tout secours et épuisés par tous les fléaux de la terre et des mers, vous voulez bien nous recueillir dans votre cité, dans votre maison.

52 Tandis que tout le reste du discours semble à peu près consacré à la question de la gratitude, selon la modalité prétéritive de l'impuissance à rendre la pareille. Si nous essayons de mettre les cellules formulaires (dans l'ordre où les analyse le canevas type) face au texte de Virgile, cela pourrait donner à peu près :

\begin{tabular}{|l|l|}
\hline $\begin{array}{l}\text { La partie III selon le canevas-type } \\
\text { de Vossius }\end{array}$ & Séquences en rapport dans l'Énéide \\
\hline $\begin{array}{l}\text { 1. «C'est au-dessus de nos forces » que } \\
\text { de rendre la pareille / « Nous en } \\
\text { sommes au plus haut point incapables, } \\
\text { vu nos faibles moyens" }\end{array}$ & $\begin{array}{l}\text { "Il n'est point en notre pouvoir, ô Reine, de vous remercier } \\
\text { dignent ; pas plus que ne le peut ce qui reste de la nation de } \\
\text { Dardanus, dispersée dans tout l'univers. » }\end{array}$ \\
\hline $\begin{array}{l}\text { 2. nous engageons } \\
\text { solennellement à être reconnaissants } \\
\text { et à nous souvenir du bienfait » }\end{array}$ & $\begin{array}{l}\text { "Tant que les fleuves porteront leurs eaux à la mer, tant que les } \\
\text { astres brilleront au firmament, je ne cesserai de proclamer votre } \\
\text { gloire, votre nom et vos louanges, quelles que soient les terres où } \\
\text { les destins m'appellent. » }\end{array}$ \\
\hline $\begin{array}{l}\text { 3. « Nous prions Dieu de répandre lui- } \\
\text { même les grâces qui conviennent. » }\end{array}$ & $\begin{array}{l}\text { « Puissent les dieux, s'il en est qui honorent la vertu, s'il est } \\
\text { quelque justice sur la terre, et s'il se trouve une conscience du } \\
\text { bien, vous récompenser dignement. » }\end{array}$ \\
\hline
\end{tabular}

53 Nous dirons que le résultat est particulièrement probant: il semblerait même que ce discours de Prince puisse fonctionner comme modèle paradigmatique pour définir ce qu'est, dans un discours de remerciement, la partie argumentant en faveur d'une promesse de gratitude; et qu'il puisse également être le référent à l'origine même des loci formulaires tels qu'ils sont résumés dans le synopsis de Vossius, en particulier le locus principal de la dénégation. Le texte de Virgile est très précisément exemplaire par sa promesse de gratitude.

Cela étant, il convient ici d'introduire quelques bémols.

1. Tout d'abord, en appliquant le modèle de Vossius, nous en restons à une analyse assez globale de la séquence dédiée à la partie précédente (l'amplification du bienfait) et nous n'entrons pas dans les raisons qui font qu'on passe d'une posture euphorique à une posture déplorative.

56 2. Nous parvenons certes à une analyse assez fine de la séquence dédiée à la gratitude, puisque nous sommes capables de la subdiviser et de la répartir en trois lieux argumentatifs répertoriés. Mais en poussant jusqu'au bout le principe de l'application (du canevas au texte), il apparaît que nous ne rendons pas compte de deux choses :

- Nous ne faisons rien d'une séquence intermédiaire - interpolée - dans la partie consacrée à la gratitude : «Ô temps heureux qui vous ont vue naître! Quelle gloire pour les auteurs de votre naissance !» 
-L'ordre des séquences proposées dans le canevas de la partie sur la gratitude n'est pas

l'ordre des séquences présent dans le texte de Virgile.

Qu'à cela ne tienne: nous pouvons aisément corriger cette partie et récrire Virgile d'après Vossius, c'est-à-dire en soumettant la délimitation et l'ordre des séquences au canevas que propose Vossius et en rejetant - comme il a lui-même montré la voie toute séquence interpolée. Ce qui donnerait à peu près ceci (en ajoutant au texte littéral quelques chevilles de transition, et en aménageant la disposition matérielle par des alinéas et des sauts de ligne) :

\begin{tabular}{|c|c|}
\hline L'Énéide séquencée & L'Énéide corrigée \\
\hline $\begin{array}{l}\text { Le voici devant vous, celui que vous cherchez } \\
\text { tous, le Troyen Énée, arraché aux flots de la } \\
\text { Libye. }\end{array}$ & \\
\hline $\begin{array}{l}\text { (I) Vous seule, Didon, prenant en pitié les grands } \\
\text { malheurs de Troie, vous voulez bien nous } \\
\text { recueillir, nous, les survivants du massacre grec, } \\
\text { dépourvus comme nous le sommes de tout } \\
\text { secours et épuisés par tous les fléaux de la terre } \\
\text { et des mers, vous voulez bien nous recueillir } \\
\text { dans votre cité, dans votre maison. }\end{array}$ & $\begin{array}{l}\text { (I) Vous seule, Didon, prenant en pitié les grands } \\
\text { malheurs de Troie, vous voulez bien nous } \\
\text { recueillir, nous, les survivants du massacre grec, } \\
\text { dépourvus comme nous le sommes de tout } \\
\text { secours et épuisés par tous les fléaux de la terre } \\
\text { et des mers, vous voulez bien nous recueillir } \\
\text { dans votre cité, dans votre maison. }\end{array}$ \\
\hline $\begin{array}{l}\text { (II) Il n'est point en notre pouvoir, ô Reine, de } \\
\text { vous remercier dignement; pas plus que ne le } \\
\text { peut ce qui reste de la nation de Dardanus, } \\
\text { dispersée dans tout l'univers. }\end{array}$ & $\begin{array}{l}\text { (II) Il n'est point en notre pouvoir, ô Reine, de } \\
\text { vous remercier dignement ; pas plus que ne le } \\
\text { peut ce qui reste de la nation de Dardanus, } \\
\text { dispersée dans tout l'univers. }\end{array}$ \\
\hline $\begin{array}{l}\text { Puissent les dieux, s'il en est qui honorent la } \\
\text { vertu, s'il est quelque justice sur la terre, et s'il } \\
\text { se trouve une conscience du bien, vous } \\
\text { récompenser dignement. }\end{array}$ & $\begin{array}{l}\text { [Mais] tant que les fleuves porteront leurs eaux à } \\
\text { la mer, tant que les montagnes couvriront les } \\
\text { vallées de leurs ombres, tant que les astres } \\
\text { brilleront au firmament, je ne cesserai de } \\
\text { proclamer votre gloire, votre nom et vos } \\
\text { louanges, quelles que soient les terres où les } \\
\text { destins m'appellent. }\end{array}$ \\
\hline $\begin{array}{l}\text { Ô temps heureux qui vous ont vue naitre ! Quelle } \\
\text { gloire pour les auteurs de votre naissance! }\end{array}$ & $\begin{array}{l}\text { [Enfin, ] puissent les dieux, s'il en est qui } \\
\text { honorent la vertu, s'il est quelque justice sur la } \\
\text { terre, et s'il se trouve une conscience du bien, } \\
\text { vous récompenser dignement! }\end{array}$ \\
\hline
\end{tabular}


Tant que les fleuves porteront leurs eaux à la mer, tant que les montagnes couvriront les vallées de leurs ombres, tant que les astres brilleront au firmament, je ne cesserai de proclamer votre gloire, votre nom et vos louanges, quelles que soient les terres où les destins m'appellent. pouvons être légitimement fiers du résultat. Si l'on considère tout d'abord la colonne de gauche (l'Énéide séquencée),

- le canevas des remerciements en forme nous a tout d'abord permis de délimiter dans le texte continu six séquences et d'en identifier quatre (les deux séquences interpolées ne sont identifiées que négativement, hors du système des remerciements) : une séquence consacrée à l'amplification des bienfaits; une séquence à la dénégation de pouvoir jamais rendre la pareille ; une séquence à la promesse de reconnaissance éternelle ; une séquence à la prière aux Dieux pour qu'ils récompensent la bienfaitrice ;

- il nous a également permis de mettre en réseau trois des quatre dernières séquences autour de l'argumentaire dédié à la gratitude ;

- il nous a enfin offert un modèle d'ordonnancement global en deux parties (légèrement déséquilibrées), d'abord l'amplification du bienfait, plus brève, et consécutivement la promesse de gratitude, plus ample.

Nous dirons que l'application méthodique du canevas type s'est avéré une opération satisfaisante en ce qu'elle a abouti à inscrire dans le texte une description assez fine et exhaustive, une mise en forme et une modélisation globalement opératoires. Que quatre perturbations locales persistent (le pathos de la pitié comme ressource de l'amplification du bienfait, les deux interpolations et l'ordre des séquences dans la seconde partie), cela n'est pas trop grave : on peut se replier sur l'idée qu'il est ainsi fait l'épreuve de la singularité d'un texte par rapport à un type ; on peut aussi en profiter pour s'exercer à produire un autre texte possible, l'Énéide corrigée, qui garderait les grâces de la poésie virgilienne et leur ajouterait les beautés méthodiques du canevas selon Vossius.

Mais le texte structuré en six séquences et modélisé sur deux des quatre parties du discours de remerciements n'est pas plus originel au fond: l'Énéide séquencée est également un texte possible, le texte qu'il a été possible de composer par la mise en œuvre réfléchie de notre compétence formalisatrice et modélisatrice, par l'application du protocole de composition tel qu'il est décrit dans l'analyse rhétorique du genre des remerciements en forme. Ce qui veut dire aussi que ce nous mettons en place au bout du compte, ce sont des hypothèses de description, des propositions méthodiques de structuration, qu'un nombre raisonnable d'éléments textuels corrobore mais qu'il est sans doute possible de réfuter (de falsifier) au regard des (quatre) éléments non intégrés.

Bref, notre mise en forme de l'Énéide est éminemment perfectible : une description plus intégrative l'emporterait haut la main, qui ne laisserait rien de côté et rendrait compte de tout. Mais il faudrait pour cela passer d'une pratique débutante de la rhétorique 
lectoriale à une pratique experte. Il faudrait être maître dans l'art de lire (de composer, de disposer). Il faudrait s'appeler, par exemple, Lauban.

\section{Lauban, le texte et son arbre}

\section{Analyse de texte : un exercice exemplaire}

Quelle forme est-il possible de donner au discours du Prince? Lauban nous invite à répondre: une forme carrée. Mais qu'est-ce qu'un texte carré? C'est un texte qui entrerait tout entier dans un système subdivisible sans déséquilibre, se répartissant en un nombre égal de colonnes et de lignes, où chaque nouvelle extension serait compensable en miroir par une extension équivalente. C'est un texte qui transformerait la linéarité de son fil en un espace quadrillé et remplacerait la succession de ses énoncés par des embranchements simultanés. C'est un texte qui superposerait à l'ordre syntagmatique de ses propositions un ordonnancement parfaitement paradigmatique.

63 Telle est alors la force de Lauban que de savoir lire ainsi, que de mettre en table le discours d'Énée, que de métamorphoser son texte en un arbre logique, figure visible de son intégration systématique.

M. Lauban, Archétypes analytiques sur le livre I de l'Énéide (1610): Table XXII, "Parties de l'entretien d'Énée et de Didon ${ }^{18}$ "

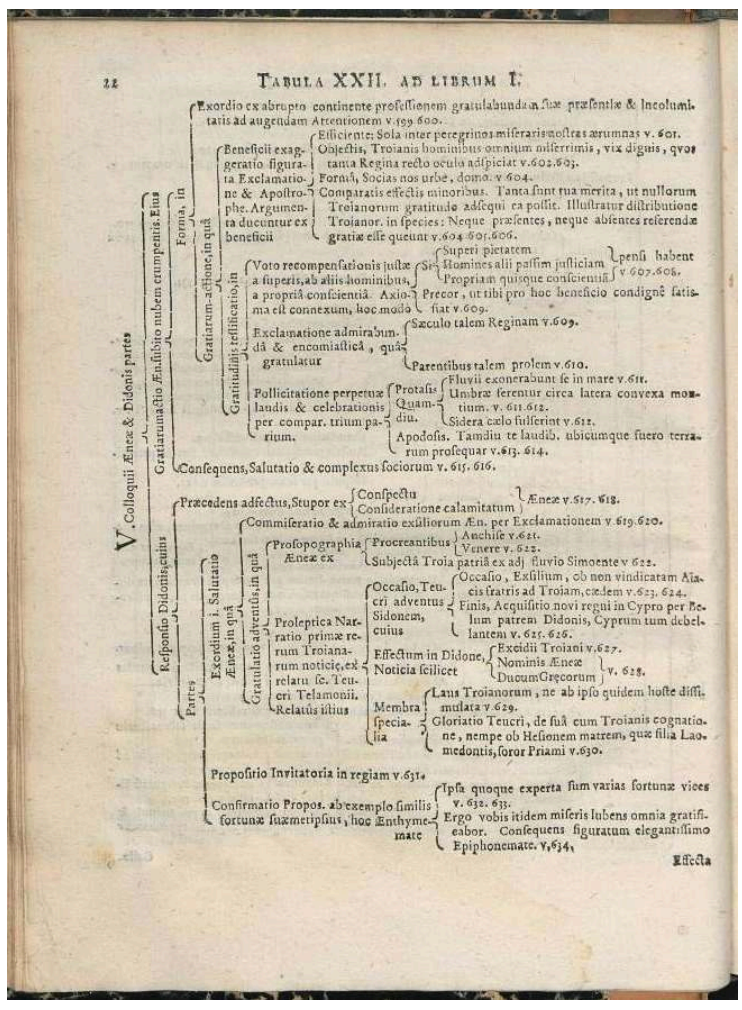

Visuellement, l'artefact produit - l'analogue rationnel pour reprendre une formule pas tout à fait inédite ${ }^{19}$ - ne se lit pas de haut en bas et ligne à ligne, mais de gauche à droite, et en suivant les branches de l'arbre, des deux fortes branches que sont, à l'extrême gauche vers le haut, « le discours de remerciement d'Énée » (« gratiarum actio 
Aen. ») et vers le bas, "la réponse de Didon» («responsio Didonis »), jusqu'aux branchioles, sur la droite, dont on constate qu'elles alignent les uns sous les autres les numéros des vers latins («v. 599.600.», «v. $601 »$, «v.602.603.», etc.), les restituant dans l'ordre du poème. Autrement dit, si l'ensemble des embranchements matérialise l'ordonnancement paradigmatique, la dernière colonne, celles des énoncés, restitue en même temps le déroulé syntagmatique.

Ce tour de force a un nom et une histoire : il s'agit là d'un tableau ramiste, autrement dit d'une classification synoptique selon une division par dichotomie inspirée des travaux sur la dialectique de Pierre de La Ramée (Ramus en latin, 1515-1572). Mais la leçon de lecture sera pour nous ailleurs. Car en entrant dans le détail des distinctions qui le structurent, on commence à comprendre qu'elles ont une double efficacité : chaque catégorie décrit un fonctionnement argumentatif et en même temps résume une séquence. Le tableau synoptique de Lauban est à la fois une décomposition du texte en séquences et une mise en réseau fonctionnel des séquences par le système des branches. Il n'est qu'à se reporter, pour s'en rendre compte, au discours que nous obtenons si nous transcrivons cursivement les indications portées au bout des accolades, en répétant ou en explicitant au besoin les formules introductives (et en en profitant, au passage, pour numéroter les séquences). 
M. Lauban, Archetyporum Analyticorum... [Archétypes analytiques...], 1610, traduction et transcription Ch. Noille

Extrait de la Table XXII, «Les parties du dialogue entre Énée et Didon »

Discours de remerciement d'Énée qui sort brusquement du nuage qui le dérobait.

$\Rightarrow$ Son plan: deux parties; à savoir l'exorde (v.595, 596); et le discours de remerciement proprement dit (v. 597-510)

$\Rightarrow$ Ses suites $:$ la salutation et les embrassades $(v .611,612)$

[SÉQUENCE 1] L'exorde est ex abrupto. Énée y porte témoignage de sa présence et de sa survie, dont il se félicite, de façon à capter l'attention v. 595-596.

Le discours de remerciement proprement dit comporte deux parties, l'amplification du bienfait (v. 97-602) et le témoignage de la gratitude (v. 603-610).

Première partie du discours de remerciement, l'amplification du bienfait prend la forme d'une exclamation et d'une apostrophe. Les arguments sont tirés :

[SÉQUENCE 2] [de la cause efficiente du bienfait : Seule parmi les étrangers, tu as eu pitié de nos misères, v. 597 ;

[SÉQUENCE 3] des récipiendaires objets du bienfait: Les Troyens, les plus malheureux des hommes, indignes qu'une telle Reine les regarde en face, v. 598-599;

[SÉQUENCE 4] de la forme du bienfait : Tu nous offres une ville, une maison, v. 600 ;

[SÉQUENCE 5] d'une comparaison du bienfait avec les effets concrets qui s'ensuivront et qui ne pourront pas être à la hauteur: Tes mérites sont tels, que la gratitude de l'ensemble des Troyens ne pourra en aucune façon y répondre. En guise d'illustration, on a une recension des espèces constituant l'ensemble en question: Ni ceux qui sont présent, ni les absents ne sont en état de témoigner de façon satisfaisante de leur reconnaissance v. 600, 601, 602 .

Seconde partie du discours de remerciement, le témoignage de la gratitude se fait en trois points : un vœu (v. 603-605), une exclamation (v. 605-606), une promesse (v. 607-610).

[SÉQUENCE 6] En témoignage de gratitude, est d'abord formulé le vœu d'une juste récompense, de la part des dieux, des autres hommes, de sa propre conscience. La proposition est liée de cette façon: Si les dieux accordent quelque valeur à la piété, si les autres hommes accordent quelque valeur à la justice, si l'on accorde quelque valeur à sa propre conscience (v. 603-604), je formule la prière que tu puisses être acquittée dignement d'un tel bienfait (v. 605).

[SÉQUENCE 7] En témoignage de gratitude est ensuite formulée une exclamation à visée d'admiration et d'éloge, dans laquelle on félicite le siècle pour une telle reine (v. 605) et les parents pour une telle descendance (v. 606).

[SÉQUENCE 8] En témoignage de gratitude est enfin formulée la promesse d'une louange et d'une célébration éternelles du bienfait, sous la forme d'une comparaison du même au même en trois points : avec, comme protase, Aussi longtemps que les fleuves se jetteront dans la mer (v. 607), que les montagnes porteront leur ombre sur les vallées (v. 607-608), que les étoiles brilleront dans le ciel (v. 608); et comme apodose : Aussi longtemps te poursuivrai-je de mes louanges, sur quelque terre que je sois (v. 609, 610).

Après le discours de remerciement, on a ce qui s'ensuit : la salutation et les embrassades des amis (v. 611, 612).

Exercices de rhétorique, 2| 2013 
Ainsi rebasculé, le propos gagne en clarté ce qu'il perd en étrangeté : nous retrouvons les gestes de séquençage et de modélisation que nous avons expérimentés en appliquant le canevas type des remerciements en forme. Mais là où, avec Vossius, nous aboutissions à six séquences (dont deux interpolées) et deux grandes parties, Lauban nous invite à remarquer huit séquences et trois parties ${ }^{20}$ :

- une séquence exordiale $\left(n^{\circ} 1\right)$, formant un préambule préparatoire ;

- une partie consacrée à l'amplification du bienfait et subdivisée du point de vue de l'elocutio en deux figures d'expression (l'exclamation et l'apostrophe) et du point de vue de l'argumentation en quatre séquences ( $\mathrm{n}^{\circ} 2$ à 5$)$;

- une partie consacrée à la promesse de gratitude et subdivisée en trois loci, en trois lieux (le lieu de la demande aux dieux, le lieu de la réjouissance, le lieu de la reconnaissance éternelle : séquences $n^{\circ} 6$ à 8 ).

$\mathrm{Au}$ jeu des ressemblances et des différences entre l'analyse rhétorique de Lauban et l'application du canevas rhétorique de Vossius, on notera que le propos du discours est identifié de la même façon (actio gratiarum, assurer de sa gratitude) et partant que sa modélisation générique reste la même (discours de remerciement); que le corps du discours est ici et là divisé en deux parties, ici et là également modélisées; mais que l'analyse intègre la séquence inaugurale et en fait une partie autonome (l'exorde); et qu'elle fait quelque chose de la séquence interpolée "Ô temps heureux, etc. ». Une plus grande couverture du texte, donc, et une plus fine granularité de sa description: puisque chaque partie est ici subdivisée en séquences parfois elles-mêmes détaillées. On peut d'ores et déjà en retenir que l'expertise de Lauban n'est pas dans l'arrangement des parties, mais dans la division des séquences et dans leur identification. Reste à entrer dans le détail de la chose.

\section{Protocole de l'analyse}

Par l'exemple, qu'est-ce que m'apprend l'exercice de Lauban, que ne m'avait pas permis de maîtriser l'application du canevas type des remerciements en forme? Trois choses complémentaires: il m'apprend à délimiter les séquences argumentatives; il m'apprend à décrire des fonctionnements argumentatifs; il m'apprend surtout à les imaginer et à les imbriquer.

La délimitation des séquences tout d'abord est éminemment dépendante de la longueur totale du texte à analyser : dans le cadre de ce discours particulièrement bref est retenu comme embrayeur de séquençage tout nouveau système propositionnel. L'énoncé de référence est en effet l'énoncé phrastique (proposition indépendante unique, propositions indépendantes coordonnées, proposition principale + subordonnées); de même qu'au niveau des subdivisions fines, l'autonomisation des éléments dans la séquence respecte leur répartition propositionnelle (voir les séquences $5,7,8$ ). Autrement dit, le séquençage de l'argumentation repose dans la plupart des cas sur des marqueurs syntaxiques. Mais pas toujours: pour les séquences $n^{\circ} 2$, 3 et 4 , la subdivision est sémantique, autonomisant chaque groupe syntagmatique (qu'il soit nominal ou verbal). Il s'agit là des quatre vers qui ouvrent l'amplification du bienfait et dont la version française que nous avons proposée s'est efforcée précisément de respecter l'ordre des arguments.

70 Ainsi délimitées selon des marqueurs syntaxiques ou sémantiques, les séquences argumentatives sont également décrites. Lauban, tout d'abord, effectue dans un certain 
nombre de cas (séquences $n^{\circ} 6,7$ et 8 du tableau ci-dessus) uniquement une description sémantique de l'argument, en en donnant, dans un énoncé formulaire synoptique, un résumé standardisé, un locus exemplaire :

-[SÉQUENCE 6] En témoignage de gratitude, est d'abord formulé le vœu d'une juste récompense, de la part des dieux, des autres hommes, de sa propre conscience. [...]

-[SÉQUENCE 7] En témoignage de gratitude est ensuite formulée une exclamation à visée d'admiration et d'éloge, dans laquelle on félicite le siècle pour une telle reine (v. 605) et les parents pour une telle descendance (v. 606). [...]

-[SÉQUENCE 8] En témoignage de gratitude est enfin formulée la promesse d'une louange et d'une célébration éternelles du bienfait [...].

Ces textus ne sont pas que des récritures minimales, ils ont bien vocation à nourrir l'outillage rhétorique, à rejoindre l'ensemble des loci argumentatifs adossé au genre des remerciements : l'énoncé formulaire est en même temps semi-formalisé, susceptible d'être mémorisé et autonomisé pour un réinvestissement ultérieur, soit qu'on le reprenne et l'imite si l'on est auteur, soit qu'on l'utilise comme un outil de remarquage et de modélisation, à l'instance des séquences types du canevas chez Vossius, en le comparant à la lettre de tel autre texte.

Mais contrairement à Vossius, Lauban ne se contente pas de cette description sémantique des loci; il en donne le plus souvent une description formelle, qui modélise le fonctionnement argumentatif de l'énoncé résumé sur un ressort type, ce qu'on nomme en rhétorique les loci communes, les ressources types de l'argumentation. Un argument en faveur d'un point à établir peut être tiré de ses causes, de ses conséquences, de ses circonstances, de ce qui lui est comparable, etc. La liste des ressources types peut varier d'un rhétoricien à l'autre, d'une école à l'autre, mais elle est extrêmement stable dans son principe : il s'agit toujours de valider un énoncé d'un point de vue rhétorique (eu égard à sa crédibilité) en considérant qu'est validé un autre énoncé en rapport (en rapport de cause, de conséquence, d'accompagnement, de comparaison...) et que le rapport en question garantit le transfert de validité. Si la cause (ou la conséquence, ou la circonstance...) d'un fait est, par exemple, exceptionnelle, il sera valide d'en conclure que le fait est exceptionnel. Ou, même chose en sens inverse, je puis établir un fait en montrant que sa cause (ou, etc.) est validée, je puis le prouver en tirant argument de sa cause (ou de, etc.).

73 Travaux pratiques. Soit l'énoncé suivant à prouver «Il s'agit d'un grand bienfait ». Vous établirez votre raisonnement en tirant argument : $a$. de sa cause ; b. de son objet ; c. de sa forme. Réponses :

-a. Est grand un bienfait que n'accorde qu'une personne. [Argument a causa efficiente]

-b. Est grand un bienfait que l'on fait à qui est dans la misère. [Argument ab objectis]

-c. Est grand un bienfait qui consiste en une faveur remarquable. [Argument $a$ forma]

74 On aura reconnu la description formelle des arguments repérés par Lauban dans les séquences 2,3 et 4 . Seulement, le lecteur rhétoricien ne fait pas tout à fait la même chose : il ne se dit pas « Je vais prouver la grandeur du bienfait par un argument tiré de la cause (de sa cause, dirions-nous), ou tiré de l'objet, ou tiré de la forme »; il se dit: «Qu'est-ce que prouve tel énoncé et comment le prouve-t-il ?» - l'énoncé en question étant très littéralement celui-ci :

Vous seule, Didon, prenant en pitié les grands malheurs de Troie, vous voulez bien nous recueillir, nous, les survivants du massacre grec, dépourvus comme nous le 
sommes de tout secours et épuisés par tous les fléaux de la terre et des mers, vous voulez bien nous recueillir dans votre cité, dans votre maison.

Autrement dit, avant que de décrire un argument, il faut l'expliciter, ou plus exactement, non seulement il faut interpréter l'énoncé comme argumentatif (prérequis définitoire de la rhétoricité, nous l'avons vu avec Vossius) et identifier de quoi il est probatoire (la grandeur du bienfait, confer ce que le canevas type de Vossius nous avait permis de structurer), mais surtout de quelle manière il argumente, dans quel raisonnement il s'intègre. C'est à ce niveau-là que les modèles que sont les ressorts types et la structure syllogistique les mobilisant permettent d'encadrer l'imagination rhétoricienne à l'œuvre. Il n'est qu'à déplier la structuration argumentative impliquée par la description de Lauban pour s'en rendre compte. Car pendant qu'Énée déclare, « Vous seule, Didon, etc. », Lauban entend donc à peu près ceci :

- a) Est grand un bienfait que n'accorde qu'une personne.

Or vous êtes la seule à nous accueillir.

Donc est grand le bienfait que vous nous accordez.

- b) Est grand un bienfait que l'on fait à qui est dans la misère.

Or nous sommes dans la misère.

Donc, etc.

- c) Est grand un bienfait qui consiste en une faveur remarquable.

Or vous nous recevez dans votre cité, dans votre maison

Donc, etc.

La lecture rhétorique s'avère sous cet angle un formidable opérateur de textualité, puisqu'elle est capable de passer de trois groupes sémantiques (groupe nominal sujet, groupe nominal complément d'objet, groupe verbal) à un logos composé et de générer un texte possible par un double processus de dépliement, du groupe syntagmatique à trois propositions conjointes (majeure, mineure, conclusion), et potentiellement (mais pas ici...) de la majeure et de la mineure à leurs propres cellules syllogistiques les établissant - ce qui donnerait, pour un énoncé du type Sujet + Verbe + Complément, un discours en trois paragraphes. Et l'on se souviendra au passage que le syllogisme n'est pas qu'une structure argumentative : il peut supporter une structure textuelle ${ }^{21}$.

Mais pour en revenir à la mise en forme à la fois syntagmatique et paradigmatique de l'énoncé virgilien, nous pouvons à présent dire en quoi consiste l'expertise du rhétoricien Lauban. Lauban mobilise un modèle syllogistique interventionniste, qui lui permet de manipuler le texte et plus précisément de le découper. Le réseau organisationnel étant posé (le canevas type des remerciements en forme, avec, selon Vossius, trois parties possibles dans tous les cas, et une dernière sous conditions), nous en étions restés à une répartition grossière des énoncés par identification sémantique (une partie dédiée à l'amplification du bienfait, une partie dédiée à la promesse de gratitude), laissant de côté un certain nombre de problèmes. Lauban ne fait pas autre chose, mais il le fait de façon plus fine. Avec l'encyclopédie qui est la sienne non seulement des partes, mais aussi des loci (des formulations types) et des loci communes (des ressources types de l'argumentation), il interroge le fil de l'énoncé pour isoler ce qui en lui peut fonctionner comme argument probatoire : et les focales qu'il introduit ainsi (ici sur un groupe nominal susceptible de fonctionner comme argument ab objectis dans l'amplification du bienfait, là sur un regroupement propositionnel susceptible de fonctionner comme lieu formulaire de la réjouissance partagée dans une visée de démonstration de reconnaissance) sont autant de repères dans le réseau 
syntagmatique, autant de séquences mises en réseau à l'intérieur de l'une ou l'autre partie. Les loci constituent ici un outillage extrêmement raffiné pour identifier (dans l'axe paradigmatique de l'argumentation) et délimiter (dans l'axe de leur extension) toutes sortes d'énoncés.

C'est ainsi que par l'expérimentation d'un outillage qui nous est globalement étranger (les parties types d'un genre de discours insoupçonné d'une part, et d'autre part ces modélisations de l'argumentaires que sont les loci formulaires et les loci formalisés), nous avons un peu progressé dans l'apprentissage des gestes et techniques de la disposition, de la mise en forme du texte.

Tout d'abord, des modèles existent donc qui peuvent nous servir de relais pour identifier des liaisons et des hiérarchies entre les séquences, des réseaux, bref une cohérence, un plan d'ensemble: ici en l'occurrence, les canevas types d'un genre discursif ; ailleurs ce pourra tout aussi bien être les canevas types du récit ou du drame - distincts des formalisations de l'intrigue et autres schémas « actantiels » en ce qu'ils rendent compte du déroulé syntagmatique de l'histoire racontée et qu'ils sont du côté de ce que les philologues allemands (avec lesquels certains des formalistes russes entreront en débat) nommaient la composition ou agencement du texte (du discours du récit, si l'on veut) en opposition à leur définition restreinte de la dispositio comme agencement (paradigmatique) de la fabula ${ }^{22}$.

Mais ces modèles nous permettent également d'identifier une séquence (qu'elle soit longue : une partie; ou brève : un locus), en introduisant dans la cursivité une logique d'attention et de remarquage, ici motivée par une interrogation sur la finalité argumentative de l'énoncé qu'on est en train de lire, ailleurs par une interrogation comparable sur la finalité dramatique, épistémique, ou autre. Car dès qu'on se demande "À quoi ça sert », on entre bel et bien dans un processus d'arrêt, de mise à distance, et partant d'autonomisation à la fois fonctionnelle et séquentielle. Ou pour le dire autrement, l'« outillage » sémantique pour modéliser l'énoncé - ici composé de loci, ailleurs de scènes ou d'épisodes - n'existe que métaphoriquement : dans les faits ce sont des hypothèses de structuration (niveau formel) qui sont motivées par des décisions herméneutiques (sur la rhétoricité de l'énoncé, sur sa narrativité, sur son didactisme...) et qui engagent l'identité fonctionnelle de la séquence. Ce qui revient à dire que méthodologiquement, le relais de dispositifs types offre une grammaire de l'identification séquentielle, qui est un auxiliaire précieux dans les processus lectoriaux de repérage et de séquençage.

81 Identifier pour séquencer, modéliser pour organiser : si la disposition est toujours une mise en forme du sémantisme (par division, par ordonnancement), sa mise en œuvre méthodique trouve dans l'encyclopédie des dispositifs types du récit ou de l'argumentation un soutien cognitif évident, qui au bout du compte en accroît l'expertise. Mais il existe aussi des batailles d'experts.

\section{Cohérence des hypothèses}

82 L'outillage des loci et des partes est à ce point fourni qu'il autorise bien sûr de multiples adaptations. In situ, en tant qu'il accompagne et relaie la lecture d'un texte, il devient un art de trouver des hypothèses de description et nourrit ainsi une pratique de la mise en forme inventive, pour ne pas dire éminemment révisable. Se pose alors la question de la pertinence des hypothèses, autrement dit la question des critères de la mise en 
forme. Deux critères ont déjà été évoqués pour qualifier l'expertise de Lauban: l'intégration de toutes les séquences du texte dans un système organisationnel; le niveau de granularité de la description.

Peut-être peut-on suggérer qu'à ce titre, le travail de Lauban sur le discours d'Énée, c'est purement et simplement un art de cacher la poussière sous le tapis : la lecture de Lauban attrape tout, justifie tout, nivelle tout - jusque dans les détails. Le discours du Prince passé à la moulinette de la mise en forme par Lauban, ce n'est plus un texte, c'est un paradigme - c'est un monument-c'est le type même du discours de remerciement, en deux parties égales et également subdivisées.

Lauban sera ici pour nous le nom du maestro qui va répondre aux questions que nous avons laissées pendantes lors de notre expérimentation du canevas type selon Vossius.

Question $n^{\circ} 1$ de l'impétrant. - Maître, à quoi sert l'énoncé initial du discours d'Énée dans des remerciements en forme?

Réponse $n^{\circ} 1 .-$ Quand Énée dit «Le voici devant vous, celui que vous cherchez tous, le Troyen Énée, arraché aux flots de la Libye », le syllogisme est le suivant :

C'est une grande chose qu'une survie miraculeuse.

Or me voici [Preuve directe établie par le témoignage].

Donc c'est une grande chose que je sois là à vous parler. Et je m'en félicite. D'où l'attention.

On dira qu'Énée démontre la grandeur (l'importance) de ce qu'il dit et l'amplifie en s'en félicitant. Rappel complémentaire : la magnitudo rei (litt., grandeur de la chose) est l'un des lieux possibles dans les dispositifs attentionnels, une des formules-standard pour déclencher l'attention, en particulier dans la partie dévolue au travail de l'écoute et de la recevabilité du discours, en amont du discours, autrement dit l'exorde.

Résumé $\mathrm{n}^{\circ} 1 .-$ «L'exorde est ex abrupto. Énée y porte témoignage de sa présence et de sa survie, dont il se félicite, de façon à capter l'attention. » (Lauban)

Question $\mathrm{n}^{\circ}$ 2. - À quoi sert, dans l'amplification des bienfaits, la séquence sur les malheurs passés des Troyens?

Réponse $\mathrm{n}^{\circ}$ 2. - Quand Énée dit «[...] vous voulez bien nous recueillir, nous, les survivants $d u$ massacre grec, dépourvus comme nous le sommes de tout secours et épuisés par tous les fléaux de la terre et des mers [...] », le syllogisme est le suivant :

Est grand un bienfait que l'on fait à qui est dans la misère.

Or nous sommes dans la misère. [= Mineure amplifiée $]$

Donc, etc.

89 Résumé $n^{\circ}$ 2. - «Les arguments sont tirés [...] des récipiendaires objets du bienfait: Les Troyens, les plus malheureux des hommes, indignes qu'une telle Reine les regarde en face, v. 598-599. » (Lauban)

Question $n^{\circ}$ 3. - Pour qu'il y ait amplification du bienfait, ne faut-il pas une partie un peu plus ample?

Réponse $\mathrm{n}^{\circ}$ 3. - L'amplification est une surenchère, dans le nombre d'arguments comme dans leur extension. Quand Énée dit : «Vous seule, Didon, etc. », il est possible de repérer non pas un, mais trois arguments, tirés de l'origine, de l'objet récipiendaire et 
de la forme du bienfait. Quand Énée ajoute «Il n'est point en notre pouvoir, ô Reine, de vous remercier dignement; pas plus que ne le peut ce qui reste de la nation de Dardanus, dispersée dans tout l'univers [...]», il est également possible de rattacher cette séquence à l'amplification du bienfait de la façon suivante :

Est grand un bienfait que l'on ne peut payer de retour. [Argument ab effectis]

Or, aucun d'entre nous ne pourra en aucune façon rendre la pareille. [= Mineure

amplifiée]

Donc, etc.

91 La Mineure est amplifiée par un argument tiré du détail de la chose (de la recension de ses parties : ab enumeratione, syn. a distributione) :

$\mathrm{Ni}$ les Troyens qui sont ici, ni les Troyens dispersés ailleurs ne sont en état de rendre la pareille.

Donc, aucun d'entre nous ne pourra en aucune façon rendre la pareille.

Ce qui fait que la première partie comportera six vers et la seconde partie sept vers et demi.

Résumé $\mathrm{n}^{\circ}$ 3. - «Amplification du bienfait. Argument tiré d'une comparaison du bienfait avec les effets concrets qui s'ensuivront et qui ne pourront pas être à la hauteur. En guise d'illustration, on a une recension des espèces constituant l'ensemble en question [...]. » (Lauban)

Question $\mathrm{n}^{\circ}$ 4. - A quoi sert l'éloge du siècle et celui des parents dans une démonstration de gratitude?

Réponse $\mathrm{n}^{\circ}$ 4. - Quand Énée dit : "Ô temps heureux qui vous ont vue naître! quelle gloire pour les auteurs de votre naissance! », le syllogisme est le suivant :

Est reconnaissant celui qui se réjouit et se félicite d'avoir un bienfaiteur.

Or je me réjouis et me félicite de votre présence. [= Mineure étayée par deux témoignages]

Donc, je suis très reconnaissant.

Le locus de la joie prouve la gratitude (Vossius dirait : « Nous signifions la joie que nous a procurée le bienfait »). La mineure n'est pas confirmée par un argument mais par un double témoignage effectif de réjouissance (l'éloge du siècle et l'éloge des aïeux).

Résumé $n^{\circ} 4 .-$ «En témoignage de gratitude est ensuite formulée une exclamation à visée d'admiration et d'éloge, dans laquelle on félicite le siècle pour une telle reine et les parents pour une telle descendance. » (Lauban)

Dernière question. - Est-il indifférent que l'ordre des séquences ne soit pas celui des remerciements en forme selon Vossius ? Est-il indifférent que le texte donne notre canevas type dans le désordre?

... Pas de réponse du côté de Lauban... Après tout, il n'est pas sûr que le canevas type de Vossius soit le meilleur des plans possibles. Peut-être le témoignage de réjouissance peut-il être intégré dans la partie sur la gratitude? Peut-être la demande faite aux dieux de payer de retour la bienfaitrice peut-elle anticiper sur la promesse de reconnaissance éternelle ?...

Lauban ne nous en apprendra pas plus. Nous en resterons devant sa table analytique avec un discours de Prince exemplaire par sa mise en forme, divisé en huit séquences et ordonné en deux parties (plus un exorde), intégralement reformulé en lieux types. Un texte parfaitement équilibré, assurément, mais par là même immobile et spatialisé, 
littéralement projeté en un tableau arborescent : sans doute aussi, donc, un texte qu'il doit être possible de remettre en mouvement.

\section{Ferrazzi, une forme en mouvement}

\section{Analyse du texte et protocole de l'analyse : les formes dysfonctionnelles} focaliser l'attention sur la grandeur de ce qui va être dit (sur son importance), il la focalise sur la personne d'Énée, en signifiant que c'est sa présence même qui est une grande chose.

101 2. La séquence 5 (« Il n'est point en notre pouvoir, ô Reine, de vous remercier, etc. ») se révèle un peu baladeuse, puisque selon le canevas type de Vossius, c'est l'une des deux formules types susceptibles d'ouvrir la partie sur la promesse de gratitude; alors que Lauban en fait l'ultime séquence de la partie précédente consacrée à l'amplification du bienfait.

102 3. Les séquences 6 et 8 ont un positionnement flottant dans le déroulé de l'argumentation en faveur de la gratitude : tantôt (dans le canevas type) la formule de reconnaissance éternelle est d'une part enchaînée à la formule d'impeachment initiale et peut d'autre part enchaîner sur une prière adressée aux dieux pour qu'ils comblent la bienfaitrice d'un bienfait comparable à celui qu'elle a rendu ; tantôt (grâce à Lauban) l'ordre inverse semble normalisé et la clôture sur la protestation de reconnaissance éternelle ne pas poser problème.

103 4. Enfin, la séquence 7 semble parfaitement interpolée entre les séquences 6 et 8 , puisque si elle se raccroche à la démonstration générale de gratitude, elle n'y travaille pas de la même façon, donnant par témoignage et par démonstration d'enthousiasme plus que par argumentation et raisonnement l'assurance de la reconnaissance.

On pourrait dire qu'à chaque fois, une forme est séquencée qui pose problème quant à son enchaînement avec ce qui suit ou ce qui précède et qui soulève par là même des incertitudes quant à son identification fonctionnelle. C'est ce que nous nommerons précisément une forme dysfonctionnelle. Et l'analyse de Marco Antonio Ferrazzi, professeur de rhétorique au séminaire de Padoue, va alors nous montrer comment il est possible de retravailler la modélisation rhétorique dans le sens de l'instabilité des formes et de leur pluralisation. 


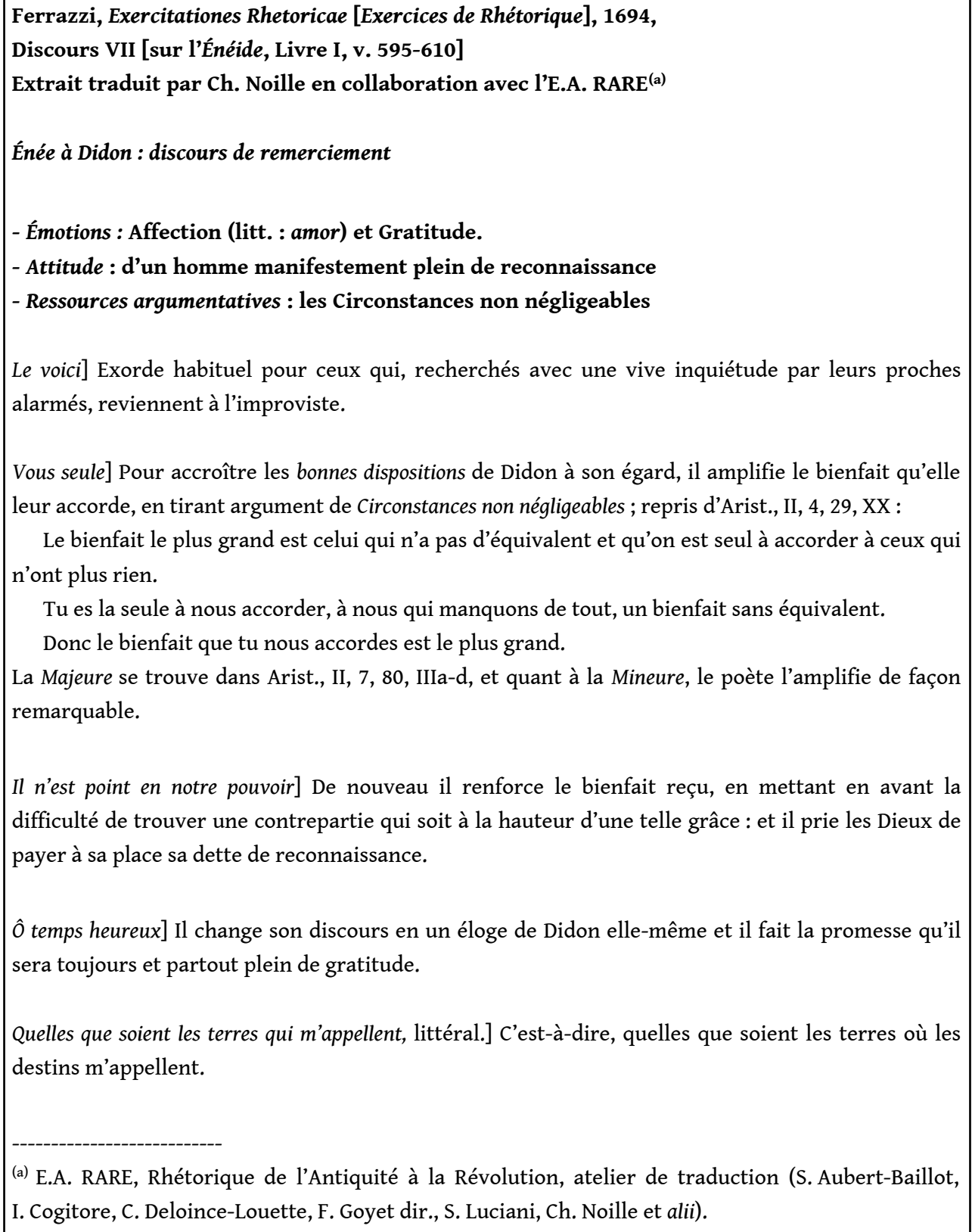

(a) E.A. RARE, Rhétorique de l'Antiquité à la Révolution, atelier de traduction (S. Aubert-Baillot, I. Cogitore, C. Deloince-Louette, F. Goyet dir., S. Luciani, Ch. Noille et alii).

Analyse méthodique, pour une lecture inquiète : pas à pas Ferrazzi marque le fil du discours d'un relevé consciencieux de ce qui va et de ce qui ne va pas. Un certain nombre de faits sont simplement mis en évidence, qui témoignent à divers titres d'un dysfonctionnement.

106 1. C'est d'abord le repérage d'un piétinement dans le mouvement général du texte : au prisme de cette lecture en effet, le discours d'Énée semble en quelque sorte bégayer, en ce qu'il redouble la partie dédiée à l'amplification du bienfait-piétinement qui entraîne alors une redite dans le commentaire : «[...] Il amplifie le bienfait qu'elle leur accorde [...]. De nouveau il renforce le bienfait reçu [...]. » L'adverbe à lui seul suffit à marquer cette idée d'une surenchère probatoire, d'un surplus, d'un excédent de preuve, dans ce qui était déjà bien établi et même amplifié dans la séquence 
précédente. Et la mise en forme du texte aboutit alors à cette disproportion flagrante, d'intégrer les deux tiers du discours dans la partie dédiée à l'amplification du bienfait.

2. C'est ensuite le repérage d'une reconversion du discours: le Prince «change son discours », dit l'analyse (en latin : « Convertit orationem... »); notation minimale d'un fait massif dans la stratégie lectoriale, le passage d'une hypothèse à l'autre concernant la proposition générale, le dessein argumentatif du texte. Tout ce qui précède en effet la séquence ô combien problématique de l'éloge ("Ô temps heureux, etc.») relève du " discours de remerciement » ainsi qu'il est précisé dès le titre synoptique que Ferrazzi donne à son analyse. Mais à partir de là s'ouvre donc un espace textuel dont le propositum n'est pas précisé et dont tout ce qu'il nous est montré est qu'il intègre un éloge, une prière et une promesse, lesquelles sont à la fois des lieux formulaires (des énoncés argumentant on ne sait en quel sens) et des figures, des formes dans lesquelles se déploie par prérequis la stratégie énonciative. Ou pour le dire autrement, les arguments formulés à la fin du texte sous la forme d'un éloge, d'une prière et d'une promesse viennent étayer une proposition discursive qui n'est plus celle des remerciements.

3. C'est enfin le repérage d'une double orientation quant aux affects que le texte mobilise. Plus précisément, dans l'en-tête que Ferrazzi récapitule pour chacune de ses analyses, on notera que figurent d'une part les ressources types exemplifiées dans l'argumentaire, et d'autre part les éléments pertinents quant à la définition du genre discursif, à savoir le résumé du titre (où le genre est parfois littéralement explicité, comme ici), l'éthos de l'orateur (mores en v.o., attitude en v.f.) et, nous y voilà, les émotions visées (ou pathos, ou motus) dont le texte fait la démonstration, dont il formule les raisons qui sont censées en être à l'origine. Or, nous dit l'analyse, le discours du Prince mobilise deux types de raisonnement pathétique, visant à la fois à la gratia et à l' amor, variante libre, chez Ferrazzi, de la captatio benevolentiae. Si la gratitude peut être rattachée sans difficulté aucune au paradigme des remerciements en forme, il n'en va pas de même de l'autre pathos que les énoncés programmeraient. La benevolentia est en effet cette bonne volonté pratique qui est corrélée en rhétorique avec ses conséquences, l'octroi d'un beneficium. Autrement dit, il est affiché dès l'en-tête que le texte ne vise pas uniquement à nourrir le cercle vertueux de la gratia, mais à établir, pour la destinataire, les raisons d'une bienveillance susceptible de se traduire en acte.

Nous pouvons ajouter que les trois faits ainsi repérés-l'hypertrophie de l'argumentaire consacré à l'amplification du bienfait, le changement dans le dessein du discours, la mention d'un affect hors système par rapport au contexte générique vont dans le même sens : ils mettent en évidence un dysfonctionnement dans la forme promue, le discours de remerciement, en focalisant l'attention sur des séquences résiduelles qui échappent au réseau paradigmatique.

Et l'on en arrive ici, en plein XVII ${ }^{\mathrm{e}}$ siècle, à la théorie des textes possibles ${ }^{23}$, ou du moins à son expérimentation dans une pratique de lecture parfaitement méthodique et formaliste. Car Ferrazzi n'est jamais prisonnier de l'hypothèse dominante de lecture selon laquelle le texte s'organise selon les formes du discours de remerciement : il est capable à la fois de mobiliser ce modèle-là de composition discursive et en même temps de le tester constamment et de le reformuler, autrement dit de le réfuter, de le mettre en concurrence avec d'autres modèles possibles. Tant et si bien que le discours qui se compose et prend forme au fur et à mesure de l'analyse est littéralement pluralisé, démultiplié en plusieurs canevas types, en une pluralité de discours fantômes qui 
hantent les remerciements en forme et les déstabilisent, travaillant tous les points de transition - et l'on ne s'étonnera pas au passage de retrouver les séquences qui, depuis notre premier relevé avec Vossius, nous posent problème, de la séquence inaugurale au tableau des misères des Troyens, de la délimitation flottante de la partie sur le bienfait au désordre des séquences conventionnelles dans la partie sur la gratitude et à l'interpolation des formes de l'éloge.

111 Premier modèle affleurant, d'extension locale, le genre de l'allocution d'arrivée, qui est mobilisé pour identifier la première séquence du discours tant dans son extension que dans sa fonction : Ferrazzi décrit en effet l'exclamation initiale (« Le voici devant vous, celui que vous cherchez tous, etc.») dans sa relation à la suite du discours, et plus précisément comme exorde, comme partie préparatoire, mais qui est censée préparer, nous est-il précisé, au type de discours que prononcent habituellement « ceux qui [...] reviennent à l'improviste ». Or, il s'agit là d'un genre répertorié, les allocutions d'arrivée, dont un des lieux formulaires exordiaux est précisément le rappel des dangers encourus et la satisfaction d'y avoir échappé. Ou comme l'explicite Vossius dans le canevas type qu'il propose ailleurs de l'allocution d'arrivée,

En premier, nous saluons les mânes de nos aïeux : et nous remercions les dieux, qu'ils nous aient reconduits sains et saufs ${ }^{24}$.

Et l'on se souvient au passage que le jésuite La Cerda avait remarqué - rendu signifiant - le changement de destinataires qui s'opère entre l'incipit et la phrase suivante ("Vous seule, Didon, etc. ») tandis que Vossius avait purement et simplement expurgé cette séquence initiale du paradigme des remerciements. La séquence initiale peut fonctionner comme exorde d'une possible allocution d'arrivée, mais elle est en même temps une interpolation dans l'argumentaire des remerciements - que n'avaient pas même réussi à conjurer complètement les prouesses intégratives de Lauban. Autre façon de dire la chose, la transition entre la première séquence et ce qui la suit s'effectue sur le mode de la bifurcation, la rejetant a posteriori du côté de l'excursus et de la digression. Ou encore : la première séquence ne prépare pas à cette suite-là. Elle exemplifie une forme - répertoriée, reconnaissable - d'un autre modèle possible de discours.

113 Autre modèle d'extension locale, et parfaitement symétrique, le genre de l'allocution de départ affleure dans la modélisation que Ferrazzi opère de la toute fin du texte. Si l'on peut à juste titre s'extasier au passage sur cet heureux temps qui semble avoir institué des genres oratoires pour toutes les circonstances du discours direct dans le récit, on rappellera qu'on n'a pas ici pour but de faire l'archéologie de ces formes institutionnelles mais d'en actualiser le savoir-faire, en mesurant l'apport de l'encyclopédie des formes rhétoriques dans la mise en forme du texte; et que pour ce faire notre présentation est obligée de prendre un biais, le moins mauvais possible, qui est celui de faire surgir un à un des éléments de l'encyclopédie, tels que les canevas d'autres discours types, pour comprendre et expérimenter comment l'outillage formel (formulaire) opère de façon extrêmement souple afin d'identifier les lieux du texte et d'en mettre en forme le déroulé. Et précisément, l'un des lieux conclusifs des allocutions de départ est ainsi présenté dans le traité qui nous sert de référent encyclopédique, la Rhétorique restreinte de Vossius :

Nous dirons que nous garderons toujours en mémoire les bienfaits que nous avons reçus ${ }^{25}$. 
114 Ainsi donc, la position conclusive de la promesse de reconnaissance éternelle n'est peut-être pas insignifiante : peut-être que l'ordre - dans un dispositif de parole tourné vers la progression et l'accumulation de l'argumentation - n'est pas anodin ; peut-être que la reconnaissance éternelle - magnifiquement amplifiée dans le discours du Prince, des rivières à la mer, des vallées vers les montagnes, des sommets jusques aux cieux - n'a pas même fonction argumentative selon qu'elle est en position ouvrante ou fermante. D'un côté en effet (dans les remerciements en forme), il semble bien qu'elle constitue la preuve première de gratitude (dans le cas où rendre la pareille est impossible); de l'autre côté (dans les allocutions de départ), elle meuble la péroraison par les ressources du pathos, permettant d'achever sur une effusion de gratitude.

115 Autrement dit, une même forme locale, selon sa position, sera susceptible d'être identifiée en corrélation avec des contextes différents et d'embrayer sur une hypothèse différente quant à l'organisation globale du texte et quant à son genre. Nous dirons qu'au même titre que l'allocution d'arrivée, le modèle de l'allocution de départ pose très clairement la question de la liaison d'une cellule argumentative avec son contexte amont et/ou aval. Ce que l'analyse rhétorique nous apprend ici, c'est à interroger et à modéliser les transitions ; c'est à penser la mise en forme non seulement en termes de division et d'ordonnancement (de séquençage et de réseau), mais en termes d'enchaînement, de suite ; c'est à réintégrer dans l'espace du canevas la dimension du temps. Où il apparaît que l'instabilité de la modélisation générique est la condition d'une dynamique - lectoriale - des formes, d'une forme du texte - expérimentée en mouvement.

116 Mais pas seulement: si les modèles de transitions et les canevas types peuvent nous aider à pluraliser la forme du texte et son identité, ils peuvent tout aussi bien nous inviter à évaluer une forme contre une autre, à mesurer leur pertinence respective et à niveler en conséquence la modélisation du texte sur la meilleure des formes possibles. Où l'on verra alors que la rhétorique s'arrête précisément là, où commence l'interprétation.

\section{Réfutabilité des hypothèses et décision herméneutique : d'un discours, l'autre.}

117 D'un bloc homogène et indifférencié, le discours du Prince est devenu peu à peu pour nous une forme en mouvement, il a cessé d'être une masse pour prendre appui sur un certain nombre de relais, pour donner figure à un certain nombre de séquences et prendre en retour la figure de ses divers réseaux. Voilà une parole assurément exemplaire, qui commence à la manière d'une allocution d'arrivée, pour devenir un discours de remerciement (à peu près) en forme, avant de s'achever comme un discours de départ. Un discours de remerciement à peu près en forme : le diable est dans les détails et à vrai dire, rien n'a été fait des remarques de Ferrazzi concernant l'introduction d'une visée pathétique hors-système (la bienveillance et son corollaire, le bienfait), ni de son diagnostic sur la rupture qu'introduit la séquence d'éloge dans le propos général des remerciements. Rien n'a été fait non plus des malheurs des Troyens, dont le tableau semble là encore interpolé dans le texte des remerciements.

118 Et rien n'a été fait enfin d'une hypothèse de Ferrazzi que nous avons jusqu'ici préféré passer sous silence et qui porte pourtant le doute jusque dans la séquence la plus 
fermement raccrochée à l'amplification du bienfait, à savoir l'interpellation initiale à Didon. Pendant qu'Énée dit en effet :

Vous seule, Didon, prenant en pitié les grands malheurs de Troie, vous voulez bien nous recueillir, nous, les survivants du massacre grec, dépourvus comme nous le sommes de tout secours et épuisés par tous les fléaux de la terre et des mers, vous voulez bien nous recueillir dans votre cité, dans votre maison.

Ferrazzi nous fait remarquer ceci :

Pour accroître les bonnes dispositions (en latin, benevolentia) de Didon à son égard, il amplifie le bienfait qu'elle leur accorde, en tirant argument de Circonstances non négligeables [...].

119 Du point de vue de son extension, la deuxième séquence délimitée par Ferrazzi rassemble dans un montage en trois éléments les trois séquences différenciées dans l'arbre analytique de Lauban, mais l'analyse argumentative revient grosso modo au même, comme le montre la restitution du syllogisme. Nous dirons que Ferrazzi s'est arrêté à l'embranchement précédent. Mais du point de vue de sa compréhension, la deuxième séquence s'avère elle aussi une forme bi-fonctionnelle, sinon dysfonctionnelle : l'argument tiré des circonstances vise certes à amplifier le bienfait mais il est également subordonné à un autre but, « accroître les bonnes dispositions de Didon à son égard». Revoilà, en plein cœur du discours, l'hypothèse activée d'un dessein argumentatif autre, lié à la benevolentia, d'une proposition générale qui pourrait bien s'appliquer également à la partie qu'isole Ferrazzi quand il marque dans le discours un tournant au niveau de la séquence d'éloge (" ô temps heureux, etc. »).

D'un côté, plusieurs séquences peuvent être mises en réseau autour du programme des remerciements en forme; de l'autre côté, un autre programme semble prendre forme et appui, sur telle séquence intermédiaire ambivalente, sur le dernier tiers du texte, sur les ressources mobilisées en vue d'un affect intéressé. Telles sont les données du problème, et l'on sent bien que la réponse est à portée de main, dans un des tiroirs de l'armoire à rhétorique; ou encore, qu'il existe quelque part un modèle rhétorique répertorié qui nous permettrait de donner à la fois identité et structure à ce qui nous échappe encore en partie, à l'autre figure du texte.

D'un coup de baguette magique, Vossius le sort de son chapeau-comme quoi sa Rhétorique restreinte n'est pas si restreinte qu'elle veut bien le dire. Cela se nomme la requête indirecte. 


\section{G. J. VOSSIUS, Rhetorices Contractae [Rhétorique Restreinte] \\ $1^{\text {re }}$ éd. 1622, Saumur, 1677, p. 208-209, traduction de Ch. Noille}

\section{Discours de requête [Extraits, nous soulignons]}

3. La requête est tantôt directe, tantôt oblique. Dans le premier cas, nous énonçons notre requête ouvertement ; dans l'autre cas, nous procédons par insinuation. [...]

8. Nous ferons usage d'une requête oblique, si ce que nous réclamons n'est pas tout à fait honnête ; ou si la personne auprès de qui nous réclamons n'est pas tout à fait un ami.

9. Dans ce cas-là, nous présenterons d'abord à quel point est douloureux le sentiment du déshonneur chez celui qui est dans le dénuement; et à quel point semble être grande l'audace qu'il y a de demander quelque chose que l'on ne mérite en aucune sorte; ou de demander à quelqu'un à tout le moins à qui l'on ne procurera en retour aucune gratitude qui soit digne du bienfait reçu.

10. Nous ajoutons que nous avons conçu cependant un immense espoir de ne pas être repoussés. Et pour ne pas sembler dire cela à la légère, nous présentons les raisons de notre confiance: comme le fait que notre interlocuteur a rendu un tel service à des personnes à qui il eût pu le refuser avec plus de justice ; et aussi, qu'il y a quelque chose de divin dans cette générosité des hommes qui prend plaisir à être utile à tous. [...]

13. Il y a place également ici pour la louange des parents et des aïeux des deux côtés : et, s'ils ont été liés par l'amitié, nous le rappellerons, ainsi que les charges officielles qu'ils ont, entre eux, favorisées et renforcées. Nous indiquerons également qu'il est beau que les héritiers des charges paternelles soient en même temps les héritiers de l'amitié.

14. Ensuite nous montrerons que notre requête ne contrevient ni à l'honnêteté, ni à la justice, ni à la piété, et qu'elle n'offre pas de difficulté à celui que nous sollicitons; mais pour nous elle est plus qu'utile, elle est nécessaire. Et nous exciterons pour cela la pitié, en tirant argument de notre propre dénuement et de notre malheur. [...]

17. Enfin nous promettrons que nous ne serons pas des ingrats et qu'aussi forte que soit notre aptitude à la gratitude, elle ne pourra être que nulle ou infime en regard de ce que nous devons; cependant nous promettrons de tendre tout notre esprit et toute notre volonté pour entretenir toujours ce sentiment. Et nous nous consacrerons tout entiers à notre devoir de loyauté à l'égard de notre interlocuteur.

Très clairement, un grand nombre d'indices plaide désormais pour modéliser le discours du Prince sur la forme de la requête oblique, celle qui procède par insinuation.

1. Les Carthaginois harcèlent des Troyens naufragés sur leurs côtes et en conséquence, peut-être que la Reine Didon "n'est pas tout à fait un[e] ami[e]» : s'il y a requête, la prudence voudrait qu'elle soit oblique. Le contexte pragmatique est cohérent avec la situation discursive en jeu. Surtout, le postulat d'inimitié place au centre du dessein argumentatif l'expurgation d'une mauvaise volonté (potentielle ou réelle) et la récupération de la fameuse benevolentia, cette bonne disposition qui vous enjoint à poursuivre dans un acte de bienfaisance - à répondre favorablement à la sollicitation et que Ferrazzi mettait en balance avec la gratia dans l'en-tête de son analyse. 
124 2. La requête oblique permet de faire un sort aux deux séquences argumentatives qui nous ont constamment résisté : l'exposition du dénuement (argument pour en appeler à la pitié) et l'éloge des parents (locus formulaire s'il en est de la captatio benevolentiae).

3. La requête oblique partage avec le discours de remerciements des énoncés formulaires tout en remotivant leur fonction argumentative: la reconnaissance de l'impuissance à rendre la pareille (argument de l'indignité), l'aveu que seules les choses les plus inouïes peuvent récompenser cette faveur (argument de la générosité sublime), la promesse de reconnaissance éternelle (lieu répertorié de toutes les formes de sollicitation, que la requête soit directe ou oblique).

126 4. La requête oblique permet enfin de faire un sort à deux des éléments vifs concernant l'ordonnancement: l'insertion médiane d'une séquence d'éloge et surtout la position ultime de la séquence de reconnaissance comme péroraison par effusion de gratitude (au même titre que dans l'allocution de départ).

127 Le modèle de la requête oblique nous fournit ainsi des éléments formels pour aider à des opérations de division, d'ordonnancement et d'enchaînement dans notre lecture du texte. Le discours du Prince peut se mouler dans la forme de la requête et en énonce souvent les formules. Mais il en manque une: l'énoncé du propositum, du dessein propositionnel global. Car si ce texte peut prendre dans la lecture la forme d'une requête, il conviendrait d'abord de savoir de quoi il énonce la requête, autrement dit en quoi consiste la demande d'Énée à Didon (et accessoirement, où elle est littéralement formulée).

128 Là encore, le zèle des rhétoriciens nous secondera à propos. Un des prérequis de la requête, c'est de solliciter une réponse - qu'il y ait ou non quelque chose d'accordé. Et nous l'avions mentionné, si le discours d'Énée est une réponse à la faveur octroyée par Didon (et à ce titre occupe la place d'un discours de remerciements), il est par ailleurs suivi lui-même d'une réponse de Didon (tant et si bien qu'il occupe la place d'une demande). Or, que répond Didon? C'est Lauban qui nous l'explique - lui qui pourtant a bouclé le discours d'Énée dans la forme d'un remerciement. 
M. Lauban, Archetyporum Analyticorum... [Archétypes analytiques...], 1610, traduction et transcription Ch. Noille

Extrait de la Table XXII, «Les parties du dialogue entre Énée et Didon »

Réponse de Didon

Dont nous avons d'abord la réaction, à savoir sa stupéfaction à la vue d'Énée et à l'écoute de ses malheurs (v. 613, 614)

Puis les parties du discours proprement dit sont: l'exorde, c'est-à-dire la salutation d'Énée [v. 615-626]; la proposition (l'invitation dans son palais: v.627); et la confirmation de la proposition par un exemple semblable (v. 628-630).

Vers 615-626 : l'exorde. La salutation d'énée est divisée en deux : d'abord la pitié et l'admiration à son égard (v. 615,616$)$; ensuite dans un mouvement de congratulation, elle se félicite de sa venue [v. 617-626]. [...] La partie de congratulation se divise en une prosopographie d'Énée (v. 617, 618) et la narration des circonstances où elle a entendu parler en premier des événements de Troie [v. 619-626]. [...] Cette narration se clôt sur l'éloge des Troyens (v. 625) et la glorification qu'en faisait même un ennemi, Teucer (v. 626). [...]

Vers 627-630 : la proposition (l'invitation) [...] ; et la confirmation de l'invitation [...].

Sur un discours de dix-huit vers, Didon en consacre quatorze selon Lauban à l'exorde, c'est-à-dire à une partie préparant très lourdement l'auditoire, avant d'entrer dans le vif du discours (qui se réduit à quatre vers). C'est typiquement là une structure dévolue à récupérer un auditoire qui est censé ne pas être dans de bonnes dispositions. Plus précisément, ce sont les misères d'Énée (et de son peuple) qui trouvent d'abord ici leur répondant dans un exorde à visée consolatoire (pitié et admiration de Didon), mais l'exorde poursuit également une visée congratulatoire: il ne faudrait pas qu'Énée puisse croire que Didon ne se félicite pas de sa présence.

Puis le discours proprement dit est l'octroi d'une faveur (d'une nouvelle faveur) : nous y voilà, Didon invite Énée dans son palais.

131 La boucle est bouclée. La réponse de Didon interprète (et permet d'interpréter) le discours d'Énée dans le sens d'une récrimination implicite (c'est là que l'absence de toute démonstration de joie, cette partie que Vossius plaçait à l'incipit des remerciements, prend tout son sens) et d'une demande formulée littéralement à la fin de la séquence « Vous seule, Didon, etc. » :

[...] Urbe, domo socias. / Vous voulez bien nous recueillir dans votre cité, dans votre maison. [Nous soulignons.]

Ce qu'Énée formule là n'est pas simple : car s'il est exact de dire que Didon accorde aux Troyens la faveur de rester en paix sur le territoire de la ville, elle ne leur a pas encore ouvert sa maison. L'énoncé " dans votre maison " pose problème. Soit l'on considère qu'il ne faut pas le prendre dans son sens littéral et qu'il faut comprendre par exemple "dans votre ville, qui est pour votre peuple et pour vous comme une maison »; soit l'on considère qu'Énée anticipe des remerciements sur une faveur qui n'est pas encore accordée - voilà la requête oblique - et qui le sera donc dans la réponse de l'intéressée : 
Vous donc, ô étrangers, venez donc vous vous reposer sous notre toit ${ }^{26} \ldots$

Ou comme l'explicitait La Cerda (on devrait toujours se méfier de ce qu'écrivent les jésuites espagnols) :

Énée amplifie la générosité de Didon [...] et la cité qu'elle leur offre, ce n'est pas encore assez, elle leur offre sa maison ${ }^{27}[. .$.$] .$

La métamorphose est ainsi opérée : on croyait avoir un discours exemplaire dans le genre des remerciements, et on se retrouve - presque - avec un discours de sollicitation: un texte qui peut en épouser la forme et qui en énonce (obliquement) l'objet. Des deux côtés, le séquençage reste le même : mais la hiérarchie et la fonction des séquences varie d'un réseau à l'autre, d'une cohérence à l'autre, de même que le sens de leur arrangement, de leur enchaînement ; et plus encore varie l'énoncé censé contenir et résumer la proposition globale, la formule livrant en quelque sorte l'identité générique du discours et constituant matériellement son centre de gravité - ici, la formule enjambant les vers 600-601 : «il n'est pas en notre pouvoir, ô Reine, de vous remercier dignement » et là le groupe verbal du début du vers 600 « vous voulez bien nous recueillir dans votre cité, dans votre maison ».

Bref, où s'arrête la description et où commence l'interprétation? Où l'analyse rhétorique cède-t-elle le pas à la reprise herméneutique du sens? Jusqu'où vont les opérations de mise en forme, de composition du texte selon les trois grands gestes que nous avons repérés et expérimentés, par division en séquences, par regroupement en parties, par transition dynamique ? Où placer le curseur entre une expérience lectoriale de formalisation et une pratique herméneutique?

Sans doute, pour une part, du côté de l'identification : qu'il s'agisse de l'identité d'un argument (quel ressort mobilise-t-il ?), de l'identité d'une partie (y a-t-il ou non une partie dévolue à la promesse de gratitude ?), ou plus encore de l'identité de l'ensemble $\mathrm{du}$ réseau et du dessein propositionnel. L'outillage rhétorique - qu'il soit lieu, partie, liaison, proposition, canevas-type - est à la fois un puissant opérateur pour marquer dans le fil du texte, dans son déroulé sémantique, des cellules syntagmatiques, des réseaux, des raccordements, bref, pour mettre en forme le texte ; et en même temps il constitue un formidable réservoir d'hypothèses modélisatrices, susceptibles d'être mobilisées pour identifier et résumer les formes, pour corréler un énoncé à une thèse, et partant les cellules à des ressources types, les réseaux à des canevas exemplaires, les raccordements à des genres, bref, les formes à des fonctionnements et la mise en forme à la fonction.

136 Autrement dit, il n'y a pas de description (de résumé, de plan...) sémantiquement neutre - et c'est très bien comme cela : car décrire n'est rien d'autre que mettre en forme les masses sémantiques $\mathrm{du}$ texte, que travailler sur l'organisation du sémantisme, sur sa composition.

137 En cela l'encyclopédie rhétorique dont on a fait l'archéologie de quelques éléments n'est pas très différente de l'encyclopédie narratologique comme de toute encyclopédie lectoriale intégrant une compétence informée par de multiples méthodologies. Toutes offrent le même modus operandi dans la perspective d'un art de lire, d'une rhétorique de la disposition - dont on a pu expérimenter et valider trois modes méthodiques de formalisation du sémantisme, de négociation de la lisibilité. Ici et là, même usage de l'outillage pour repérer et remarquer; même réservoir d'hypothèses génériques et fonctionnelles pour identifier et modéliser. 
138 Et même possibilité, ici et là, de retarder - artificiellement, méthodiquement, de façon experte - l'ancrage herméneutique du texte dans un niveau de sens stabilisé, de prolonger et enrichir l'étape de la description en s'aidant d'une rhétorique de la disposition, de façon à ne pas en rester à un seul système de modélisation mais à susciter une pluralité de formes possibles, une pluralité d'hypothèses modélisatrices.

139 L'interprétation se déploie où l'expérimentation formalisatrice cède le pas à la décision, où l'on n'en reste pas à l'étape technique du relevé méthodique et exhaustif de tous les embrayeurs possibles de séquençage, de cohésion, de transition dans la mise en forme du sémantisme; mais où on bascule dans le choix et la prévalence d'un système de modélisation sur un autre. Où, par exemple, le discours du Prince cesse d'être à la fois des remerciements en forme, une sollicitation oblique et quelques autres possibles, allocution d'arrivée, récrimination, éloge..., pour être actualisé, interprété, monumentalisé - sous le masque en or de l'exemplarité.

\section{Excipit}

Dans Tlön comme dans notre monde (de textes et de débats), il arrive donc que les choses se dédoublent et semblent se répéter. On nous apprend aussi qu'elles ont une certaine propension à s'effacer et à perdre leurs détails quand les gens les oublient. « Classique est l'exemple du seuil qui subsista tant qu'un mendiant s'y rendît ${ }^{28}$... »

141 - Quant à moi désormais, tant que les fleuves portent leurs eaux à la mer et que les montagnes couvrent les vallées de leurs ombres, je ne demande rien, que m'appuyer sur le seuil de l'édifice que nous sommes quelques-uns à mettre en forme et chaque jour venir un peu m'y poser : de sorte qu'irrésistiblement, on ne puisse non plus le perdre de vue.

\section{NOTES}

1. Souvenirs littéraux de Cl. Lévi-Strauss, Tristes Tropiques, Paris, Plon, 1955, chap. 1, incipit (rééd. 1984, p. 9).

2. Mes recherches sont en étroit dialogue avec deux séries de travaux sur la technique et la théorie de la disposition: d'une part ceux qui sont menés dans le cadre de l'équipe de recherche RARE - Rhétorique de l'Antiquité à la Révolution (dir. Fr. Goyet, Stendhal Grenoble 3); et d'autre part, ceux que poursuit Michel Charles depuis son Introduction à l'étude des textes (Éditions du Seuil, "Poétique ", 1995) et qu'il a entrepris de reformuler récemment, en particulier dans «Trois hypothèses pour l'analyse, avec un exemple », Poétique 164, nov. 2010, p. 387-417.

3. Souvenir de J. L. Borges, «Tlön, Uqbar, Orbis Tertius» dans Fictions (1956), Paris, Gallimard, 1957, rééd. 1981, p. 49.

4. Énéide, I, 597-610. Nous traduisons librement, d'après les traductions de Desfontaines (Paris, Quillot, 1743), Villenave (Paris, Panckoucke, 1831), M.-A. Buxus et J. Poucet (éd. en ligne L'Énéide Louvaniste, 1998), P. Veyne (Paris, Albin Michel/Les Belles Lettres, 2012). 
5. Nous nous permettons de renvoyer ici à la présentation que nous avons faite des commentaires rhétoriques dans nos travaux et aux références bibliographiques que nous y avons données : voir en particulier « Le commentaire rhétorique classique, un modèle de micro-lecture non herméneutique », dans Fabula-LHT n ${ }^{\circ} 3,1^{\text {er }}$ sept. 2007, <www.fabula.org/lht/3/Noille.html> ; ou encore « Des traités aux exercices. La gymnastique rhétorique et l'étude classique des textes ", C.A.I.E.F. $\mathrm{n}^{\circ} 61,2009$, p. 233-251.

6. Sur une approche de la lisibilité comme maitrise de la longueur textuelle par ponctuation sémantique et repérage, et sur une approche conjointe de l'illisible comme péril lié à la confusion et à l'absence de repères, voir notre analyse de cas dans $\mathrm{Ch}$. Noille, «Les lettres de Sévigné sontelles informes ? Éléments pour une rhétorique de la disposition ", Lectures de Madame de Sévigné: lettres de l'année 1671, dir. C. Lignereux, Rennes, P.U.R., 2012, en particulier les sections intitulées : «Infirmité/informité : les contours d'un problème de forme » et « Lisibilité de la lettre et gestion de l'étendue textuelle ».

7. Pour une première formulation de ces propositions, voir ibid., les sections intitulées: «Rhétorique (lectoriale) de la disposition. Une théorie de la mise en forme » et « La forme du texte : trois gestions possibles de la dispositio ». Pour une mise en parallèle avec celles de Michel Charles dans "Trois hypothèses pour l'analyse, avec un exemple» (op.cit.), voir notre compterendu dans "Sur un exemple de Michel Charles ou comment composer (avec) des textes", Poétique 169, févr. 2012, p. 97-115.

8. Sur la notion de type de discours et sur la recension des types de discours - les listes pouvant aller d'une dizaine à une vingtaine de types (systématiquement décrits dans les anciennes rhétoriques par leur canevas, leur contexte pragmatique et leurs référents exemplaires) -, voir F. Goyet, «Le problème de la typologie des discours », dans Exercices de rhétorique, 1|2013, mise en ligne $4^{\mathrm{e}}$ trim. 2013.

9. Sur les figures (en particulier les figures de pensée) comme blocs élémentaires opérant dans la structuration textuelle, voir F. Goyet, « Le recueil de sonnets comme ensemble de tableaux, c'està-dire de loci ", Programme et invention dans l'art de la Renaissance, éd. M. Hochmann, J. Kliemann, J. Koering et P. Morel, Paris, Somogy éditions d'art/Rome, Académie de France, 2008, p. 177-202 ; voir également notre article « Rhétorique de la périphrase : la figure comme dispositif de mise en forme ", dans J.-C. Cavallin et J.-D. Mazaré dir., Le Mot propre et la périphrase, Éds. Classiques Garnier, à paraître en 2013.

10. Références complètes : G.-J. Vossius, Gerardi Joannis Vossi Rhetorices Contractae, sive Partitionum Oratoriarum Libri V [Les cinq Livres De La Rhétorique Restreinte ou Des Partitions Oratoires], $1^{\text {re }}$ éd. Leyde 1621, Salmurii, Apud Renatum Pean, 1677, « XIX. De gratiarum actione, oratione gratulatoria \& lamentatoria» [ Discours de remerciements, de félicitations et de lamentations»], p. $172 \mathrm{sq}$., extraits traduits par Ch. Noille ; M. Lauban, M. Laubani archetyporum analyticorum, Liber Primus ad AEneidos Primum [Livre premier des archétypes analytiques du Livre I de l'Énéide], 1610, Table XXII, «Les parties du dialogue entre Énée et Didon », extraits traduits par Ch. Noille; M. A. Ferrazzi, Exercitationes Rhetoricae in praecipuas P. Virgilii Maronis orationes, quae in Aeneidum libris leguntur [Exercices de rhétorique sur les principaux discours de Virgile, que l'on trouve dans l'Énéide], Presses du Séminaire [Manfrè], Padoue, 1694, discours VII, extrait traduit par Ch. Noille en collaboration avec l'atelier de traduction de l'équipe RARE (S. Aubert-Baillot, I. Cogitore, C. Deloince-Louette, F. Goyet dir., S. Luciani et alii).

11. Voir P. Valéry, "Première leçon du cours de poétique " (déc. 1937), dans CEuvres, Paris, Gallimard, «La Pléiade », t. I, 1957, p. 1346 : «Tout jugement qui annonce une relation à trois termes, entre le producteur, l'œuvre et le consommateur [...] est un jugement illusoire (...). Nous ne pouvons considérer que la relation de l'œuvre à son producteur, ou bien la relation de l'œuvre à celui qu'elle modifie une fois faite. L'action du premier et la réaction du second ne peuvent jamais se confondre. Les idées que l'un et l'autre se font de l'ouvrage sont incompatibles. » Voir 
aussi U. Eco, Lector in fabula, trad. M. Bouzaher, Paris, Grasset, 1979 ; ou R. Baroni, « Entretien. À propos de La Tension narrative, Paris, Seuil, 2007 », en ligne sur le site www.vox-poetica.org.

12. Restreinte, cette rhétorique l'est en effet par rapport à un autre traité de Vossius, les Gerardi Ioannis Vossi Commentariorum Rhetoricorum sive Oratoriarum Institutionum Libri VI [Les six Livres Des Commentaires Rhétoriques ou Des Institutions Oratoires], Lugduni Batavorum, Ex Officina I. Maire,

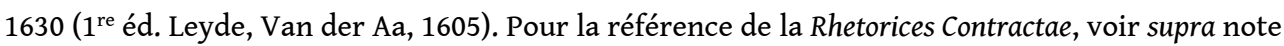
10, op. cit.

13. Propositum: proposition, dessein, propos. Partes: parties. Loci: lieux (ressources) de l'argumentation.

14. Gratia : à la fois la bonne grâce qui fait qu'on accorde un beneficium, une faveur (sens actif) et la gratitude en retour (sens réactif).

15. Sur l'apport cognitif comparé des régimes de technicité, voir P. Rabardel, Les hommes et les technologies. Une approche cognitive des instruments contemporains, Paris, Armand Colin, 1995.

16. Bien évidemment, ce ne sont pas les formules qui font l'incipit (ou en tout cas pas toujours, ou pas uniquement), mais tout autant la situation de commencement qui oriente une identification et une interprétation des formules dans le sens d'une des fonctionnalités liées à l'incipit.

17. J. L. de La Cerda, P. Virgilii Maronis Aeneidos Libri Sex Priores. Argumentis, Explicationibus et Notis illustrata, Coloniae Agrippinae, 1628, p. 110, trad. Ch. Noille. C'est précisément en nous fondant sur cette remarque que nous avons distingué un « vous » collectif dans «[...] quem quaeritis» et que nous l'avons explicité en rajoutant le pronom «tous " aux traductions littérales des XIX et $\mathrm{xx}^{\mathrm{e}}$ siècles (ce qui donne dans notre version de l'Énéide: «[...] que vous recherchez tous »), et que nous avons ajouté un terme d'appui pour expliciter l'interpellation latine "Ô sola» en "Vous seule, Didon ", reprenant en cela des traductions du XvIII ${ }^{\mathrm{e}}$ siècle.

18. Staatsbibliothek $\mathrm{zu}$ Berlin (StaBi), <http://content.staatsbibliothek-berlin.de/dms/ 598772251/800/0/00000044.jpg>.

19. Voir A. G. Baumgarten sur l'« analogon rationis », Aesthetica (1750-1758), Prolegomena, § 1, éd. Hans Rudolf Schweizer, Hambourg, Meiner, 1983 ; et surtout M. Charles, Introduction à l'étude des textes, op. cit., p. 211 : «L'analogue rationnel ressemble à un modèle, est une sorte de modèle [...]. Il est [...] un objet construit, produit, écrit par l'analyste. [...] De fait, le texte est un objet trop dense, trop complexe, les dynamiques dont j'ai esquissé la description sont, par définition, trop fuyantes, les jeux d'équilibres et de déséquilibres trop instables pour qu'on puisse envisager de les manier en l'état. [...] Il convient donc d'en venir à l'idée d'un modèle, en effet simplifié et comme "réduit", mais qui garderait ou, mieux, mettrait en évidence le régime de lecture particulier auquel tel texte ou tel ensemble de textes nous astreint. »

20. Dans les embranchements, Lauban intègre systématiquement les parties narratives interséquentielles, comme ici la séquence narrative des embrassades qui succède au discours, dont il fait une conséquence tout autant qu'une suite ("consequentes»). On pourrait modéliser alors cette séquence d'effusion gestuelle sur la péroraison, cette partie conclusive dédiée au pathos, et l'intégrer à ce titre dans le dispositif discursif, ce qui nous ferait en tout neuf séquences (et quatre parties). C'est là une complication possible du système - dans la logique de Lauban, et juste un peu au-delà.

21. Pour une analyse syllogistique de la forme de l'essai chez Montaigne, voir Déborah Knop, La cryptique chez Montaigne, doctorat, Univ. Grenoble-Alpes, 2012.

22. Pour une recension de ce débat, voir L. Doležel, Occidental Poetics. Tradition and Progress, Lincoln and London, University of Nebraska Press, 1990, «Chap. VI. Formalist Poetics : From Germany to Russia ", p.124-146. Pour son actualisation formaliste, voir par exemple B. Tomachevski, "Thématique ", dans T. Todorov, Théorie de la littérature, Paris, Seuil, 1965, p. 263-307. 
23. Pour une présentation de la théorie des textes possibles, voir la section "Textes possibles» dans l'Atelier Fabula (<www.fabula.org>), dont notre contribution, «L'affaire des textes possibles. (Enquête/Inédit)»(<www.fabula.org/atelier.php?Affaire_des_textes_possibles>).

24. G. J. Vossius, Rhetorices Contractae..., op.cit., ch. XX [« Allocutions de départ et d'arrivée »], extraits traduits par Ch. Noille, § 8, p. 179.

25. Ibid., § 5, p. 179.

26. Énéide, I, v. 627.

27. J. L. de La Cerda, op. cit., «Explicatio », p. 110. Nous traduisons.

28. J. L. Borges, « Tlön, Uqbar, Orbis Tertius », op. cit., p. 49.

\section{AUTEUR}

\section{CHRISTINE NOILLE}

Université Grenoble Alpes, RARE - Rhétorique de l'Antiquité à la Révolution 